The Astrophysical Journal, 665:1004-1022, 2007 August 20

(C) 2007. The American Astronomical Society. All rights reserved. Printed in U.S.A.

\title{
THE X-RAY PROPERTIES OF THE MOST LUMINOUS QUASARS FROM THE SLOAN DIGITAL SKY SURVEY
}

\author{
D. W. Just, ${ }^{1}$ W. N. Brandt,${ }^{1}$ O. Shemmer,${ }^{1}$ A. T. Steffen, ${ }^{1}$ D. P. Schneider,${ }^{1}$ \\ G. Chartas, ${ }^{1}$ and G. P. Garmire ${ }^{1}$ \\ Received 2007 February 13; accepted 2007 May 18
}

\begin{abstract}
Utilizing 21 new Chandra observations as well as archival Chandra, ROSAT, and XMM-Newton data, we study the $\mathrm{X}$-ray properties of a representative sample of 59 of the most optically luminous quasars in the universe $\left(M_{i} \approx-29.3\right.$ to -30.2 ) spanning a redshift range of $z \approx 1.5-4.5$. Our full sample consists of 32 quasars from the Sloan Digital Sky Survey (SDSS) Data Release 3 (DR3) quasar catalog, two additional objects in the DR3 area that were missed by the SDSS selection criteria, and 25 comparably luminous quasars at $z \gtrsim 4$. This is the largest X-ray study of such luminous quasars to date. By jointly fitting the X-ray spectra of our sample quasars, excluding radio-loud and broad absorption line (BAL) objects, we find a mean X-ray power-law photon index of $\Gamma=1.92_{-0.08}^{+0.09}$ and constrain any neutral intrinsic absorbing material to have a mean column density of $N_{\mathrm{H}} \lesssim 2 \times 10^{21} \mathrm{~cm}^{-2}$. We find, consistent with other studies, that $\Gamma$ does not change with redshift, and we constrain the amount of allowed $\Gamma$ evolution for the most luminous quasars. Our sample, excluding radio-loud and BAL quasars, has a mean X-ray-to-optical spectral slope of $\alpha_{\mathrm{ox}}=-1.80 \pm 0.02$, as well as no significant evolution of $\alpha_{\mathrm{ox}}$ with redshift. We also comment on the X-ray properties of a number of notable quasars, including an X-ray-weak quasar with several strong narrow absorption line systems, a mildly radio-loud BAL quasar, and a well-studied gravitationally lensed quasar.
\end{abstract}

Subject headings: galaxies: active — galaxies: evolution — galaxies: nuclei — methods: statistical

\section{INTRODUCTION}

The most optically luminous known quasars $\left(M_{i}<-29\right)$ serve as valuable astrophysical probes of extreme accretion conditions and the distant universe. These objects have been found to date at $z \approx 1.5-4.5$; their resulting $i$ magnitudes of $\approx 15-18$ and relatively bright multiwavelength fluxes allow them to be studied effectively with a variety of facilities across the electromagnetic spectrum. Even if they are radiating near the Eddington limit with $L / L_{\text {Edd }} \approx 1$, their energy outputs require $\approx 10^{9}-10^{10} M_{\odot}$ nuclear black holes and thus they are presumably associated with the most massive galaxies; today many of these objects have likely evolved into supergiant ellipticals found in the cores of rich clusters. As the most luminous, nontransient emitters at high redshift, these quasars have been useful in cosmological studies including measuring absorption lines from intervening line-of-sight material (e.g., Rauch 1998; Wolfe et al. 2005 and references therein), assessing the cold dark matter cosmogony (e.g., Efstathiou \& Rees 1988; Turner 1991; Springel et al. 2005), and constraining the accretion history of the universe (e.g., Croom et al. 2004; Richards et al. 2006).

The Sloan Digital Sky Survey (SDSS; York et al. 2000) is now providing the most complete selection of highly luminous quasars to date (e.g., Schneider et al. 2005, hereafter S05). A large fraction of these objects, about two-thirds, lack pointed or serendipitous X-ray detections (aside from at $z>4$, where pointed X-ray observations have detected a large fraction; e.g., Vignali et al. 2003, 2005). Accordingly, we have started a project aimed at improving understanding of the X-ray properties of the most luminous known quasars over as broad a redshift range as possible. The X-ray emission from quasars probes the innermost regions of their accretion-disk coronae where any changes in the mode of accretion might be most evident, and X-ray spectroscopy provides constraints on intrinsic and intervening absorption.

\footnotetext{
1 Department of Astronomy and Astrophysics, 525 Davey Laboratory, Pennsylvania State University, University Park, PA 16802.
}

Recent studies of the X-ray spectra (e.g., Page et al. 2005; Shemmer et al. 2005b, 2006b; Vignali et al. 2005) and X-rayto-optical spectral energy distributions (SEDs; e.g., Strateva et al. 2005; Steffen et al. 2006, hereafter S06) of quasars have generally shown no clear changes with redshift, although some exceptions have been found (e.g., Grupe et al. 2006; Kelly et al. 2007) and at lower luminosities X-ray spectral evolution may be observed (Dai et al. 2004). These results indicate that the inner regions of quasars are largely insensitive to the enormous changes in largescale cosmic environment occurring over the history of the universe. There is evidence, however, that the photon index $(\Gamma)$ of the $\mathrm{X}$-ray power-law spectrum increases as $L / L_{\text {Edd }}$ increases (e.g., Shemmer et al. 2006a) and that the X-ray-to-optical flux ratio $\left(\alpha_{\text {ox }}\right.$; Tananbaum et al. 1979) drops with increasing luminosity. Studies of the luminosity and redshift dependence of $\Gamma$ and $\alpha_{\mathrm{ox}}$ benefit from the widest possible sampling of the luminosity-redshift plane; such wide coverage is needed to break the luminosity-redshift degeneracy invariably present in flux-limited samples. By systematically studying the most luminous quasars over the full redshift range where they exist in the universe, $z \approx 1.5-4.5$, it is possible to populate one important region of this plane, complementing efforts to fill other regions of luminosity-redshift space (e.g., S06). Systematic X-ray measurements of the most luminous quasars also serve to broaden the well-sampled luminosity range available for study and thereby minimize the possibility of confusion by spurious correlations (e.g., Yuan et al. 1998).

In this paper we study, using a combination of new Chandra "snapshot" observations as well as archival Chandra, ROSAT, and XMM-Newton data, the basic X-ray properties of 32 of the 33 most luminous quasars in the SDSS Data Release 3 (DR3) quasar catalog $(\mathrm{S} 05 ; \mathrm{see} \S 2.1 .1$ for a discussion of the one quasar that is not included in our study). All 32 of the quasars in our SDSS sample have X-ray detections. We also include two comparably luminous quasars missed by the SDSS selection and an additional 25 comparably luminous non-DR3 quasars at $z \gtrsim 4$. We use our results to strengthen constraints on the $\mathrm{X}$-ray spectral and 
X-ray-to-optical SED properties of the most luminous quasars, via a combination of single-object and multiple-object analyses.

We detail the general properties of our sample in $\S 2$, as well as the X-ray observations and data reduction. X-ray, optical, and radio properties are presented in $\S 3$, and optical spectra and notes on exceptional objects appear in $\S 4$. Data analysis and results are given in $\S 5$, and a summary of our findings is given in $\S 6$. We adopt a cosmology with $H_{0}=70 \mathrm{~km} \mathrm{~s}^{-1} \mathrm{Mpc}^{-1}, \Omega_{M}=$ 0.3 , and $\Omega_{\Lambda}=0.7$.

\section{SAMPLE AND X-RAY DATA}

\subsection{Sample Selection and Properties}

\subsubsection{SDSS DR3 Quasars}

About half of our sample of highly luminous quasars has been drawn from the SDSS DR3 quasar catalog (S05). The SDSS, an optical imaging and spectroscopic survey that aims to cover about one-quarter of the entire sky, targets active galaxies for follow-up spectroscopy primarily based on their ugriz (Fukugita et al. 1996) colors and magnitudes (e.g., Richards et al. 2002). Active-galaxy candidates at $z \lesssim 3$ are spectroscopically targeted if their $i$ magnitudes are 15-19.1; high-redshift candidates are targeted if $i=15-20.2$ (the limit at $i=15$ is imposed to avoid saturation and fiber cross-talk problems in the SDSS spectroscopic observations). The DR3 quasar catalog has been constructed from SDSS spectroscopic observations over a solid angle of $4188 \mathrm{deg}^{2}$ (about 10\% of the sky). Given the large areal coverage, this catalog should contain representative members of the population of the most optically luminous quasars in the universe; i.e., other surveys are unlikely to find a population of quasars significantly more luminous than those studied here. ${ }^{2}$ About $60 \%$ of the most optically luminous quasars in the SDSS DR3 quasar catalog had been discovered in earlier surveys, such as the Hamburg Quasar Survey (e.g., Hagen et al. 1999), the Second Byurakan Survey (e.g., Stepanian et al. 2001), the University of Michigan Survey (e.g., MacAlpine \& Lewis 1978), and the Palomar Digital Sky Survey (DPOSS; e.g., Djorgovski et al. 1998).

We sorted the DR3 quasar catalog on $M_{i}$ and considered the 33 most luminous quasars in the catalog for Chandra targeting (see Fig. 1). The number 33 was chosen based on practical X-ray observing time considerations, and this sample size is large enough to provide statistically meaningful results. Of the 33 most luminous quasars, 11 already had detections in archival X-ray data and were not targeted; these archival data have been utilized in our study. The remaining 22 quasars were proposed via the Chandra Cycle 7 Guaranteed Time Observing program, and 21 of them were awarded observing time. One of our targets, SDSS J100711.81+ 053208.9, was awarded to another Chandra observer (S. F. Anderson) as part of a program studying bright and extreme broad absorption line (BAL) quasars. We do not consider the omission of SDSS J1007+0532 from our sample to be statistically problematic. In fact, owing to its BAL-quasar nature, this object would need to be removed from most of our analyses of $\alpha_{\text {ox }}, \Gamma$, and other properties in any case. Our SDSS sample thus includes 32 quasars with $M_{i}$ values of -29.28 to -30.24 , all of which have sensitive X-ray coverage; we adopt $M_{i}=-29.28$ as a practical minimum luminosity for our sample. These 32 quasars span essentially the entire range of redshift $(z \approx 1.5-4.5)$ over which such luminous objects are known, although the source statistics at $z>4$ are limited.

\footnotetext{
2 The recently released SDSS DR5 quasar catalog (Schneider et al. 2007), covering $5740 \mathrm{deg}^{2}$, further supports this assertion.
}

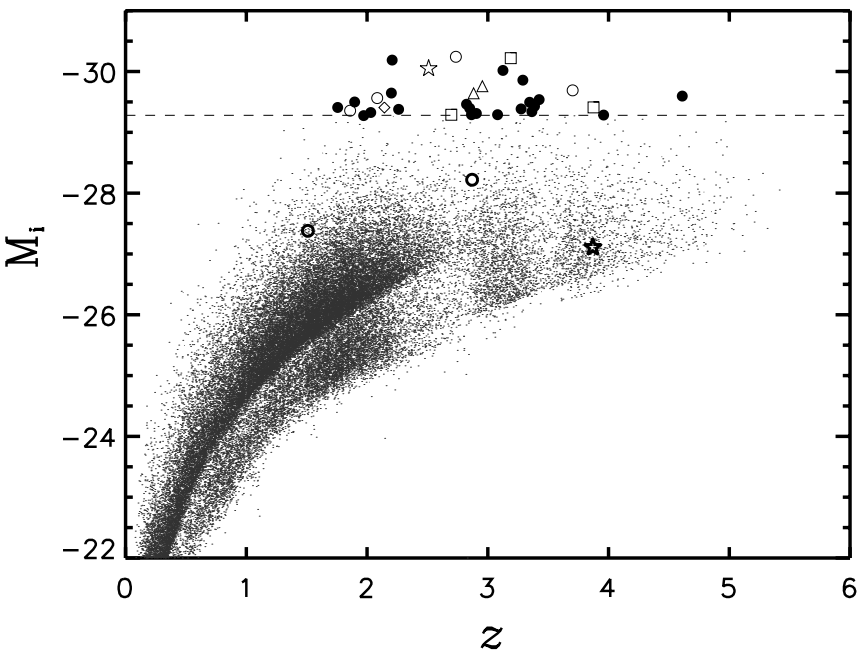

FIG. 1.-Absolute $i$-band magnitude vs. redshift for our SDSS sample compared with the SDSS DR3 quasar catalog. Our SDSS sample of 32 quasars includes both archival (open symbols) and targeted ( filled symbols) sources with Chandra (circles), XMM-Newton (triangles), and ROSAT (squares) observations. The two additional sources that were missed by the SDSS (APM 08279+5255 and HS $1603+3820$; see $\S 2.1 .2$ ) are shown as stars. The gravitationally lensed quasars APM 08279+5255, SDSS J0145-0945, and SDSS J0813+2545 have been deamplified to their true $M_{i}$ magnitudes and have bold symbols for clarity; all fail our cutoff at $M_{i}<-29.28$, which is shown as a dashed line. SDSS J1007+0532, which has been targeted by S. F. Anderson and thus needed to be removed from our sample (see $\S 2.1 .1$ ), is shown as an open diamond. Small dots represent the $\approx 46,000$ quasars in the DR3 catalog.

\subsubsection{Incompleteness and Complementary $z \gtrsim 4$ Quasars}

For the highly luminous and optically bright broad-line quasars under consideration here, the SDSS is not expected to suffer from substantial incompleteness biases. However, some incompleteness is expected due to the SDSS spectroscopic limit of $i=15$ and the fact that, at $z \approx 2.5-2.9$ and $z \approx 3.4-3.6$, the SDSS colors of quasars intersect the stellar locus (e.g., Richards et al. 2002, 2006; S05). Furthermore, about $5 \%$ of quasars are expected to be missed by the SDSS, largely due to image defects and source blending (Vanden Berk et al. 2005). We have searched for missed quasars more luminous than $M_{i}=-29.28$ in the area covered by the DR3 quasar catalog using the NASA Extragalactic Database $^{3}$ (NED) combined with accurate SDSS photometry (since the photometry in NED is not uniform and can contain significant errors). We have found only two missed quasars: APM $08279+5255$ at $z=3.91$ (e.g., Irwin et al. 1998) and HS 1603+ 3820 at $z=2.51$ (e.g., Dobrzycki et al. 1999). ${ }^{4}$ APM $08279+$ 5255 is a gravitationally lensed BAL quasar with $i=14.9$ that slightly violated the SDSS spectroscopic limit. HS 1603+3820 has a very rich spectrum of associated heavy element absorbers (e.g., Misawa et al. 2005); it is near the redshift range where the SDSS colors of quasars intersect the stellar locus and was missed by the SDSS quasar color-selection algorithm by just $0.02 \mathrm{mag}$ in $u$ (G. T. Richards 2006, private communication). Fortunately, both APM $08279+5255$ (Chartas et al. 2002) and HS 1603+3820 (PI: Dobrzycki) have X-ray detections in archival pointed Chandra

\footnotetext{
${ }^{3}$ See http://nedwww.ipac.caltech.edu.

4 Our NED search also uncovered the object QUEST J150724.0-020212.8 in the SDSS area, which is identified as an exceptionally luminous $z=1.09$ quasar by Rengstorf et al. (2004). However, that identification relied on the assumption that the single feature seen in a spectrum was the $\mathrm{Mg}$ II line. Given this tenuous identification we obtained a spectrum of this source using the Low Resolution Spectrograph (LRS) on the Hobby-Eberly Telescope (HET; Ramsey et al. 1998). Our spectrum does not show the emission feature seen by Rengstorf et al., and we identify this object as a Galactic star and not a quasar.
} 
TABLE 1

X-Ray Observation Log of the Core Sample

\begin{tabular}{|c|c|c|c|c|c|c|}
\hline Object & $z$ & $\begin{array}{c}\text { X-Ray } \\
\text { Observation } \\
\text { Date }\end{array}$ & $\begin{array}{l}\text { Chandra } \\
\text { Cycle } \\
\text { Number }\end{array}$ & $\begin{array}{c}\text { Exposure } \\
\text { Time }^{\mathrm{a}} \\
(\mathrm{ks})\end{array}$ & $\begin{array}{c}\text { Alternate } \\
\text { Designation }\end{array}$ & Notes $^{\mathrm{b}}$ \\
\hline \multicolumn{7}{|l|}{ SDSS J: } \\
\hline 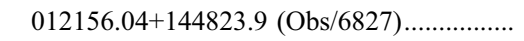 & 2.87 & 2006 Jun 21 & 7 & 3.9 & HS $0119+1432$ & $\mathrm{PC}$ \\
\hline 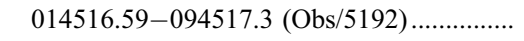 & 2.73 & 2004 Aug 23 & 5 & 13.3 & UM 673 & $\mathrm{AC}$, lensed \\
\hline 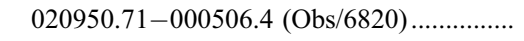 & 2.85 & 2005 Dec 02 & 7 & 2.2 & UM 402 & $\mathrm{PC}$ \\
\hline 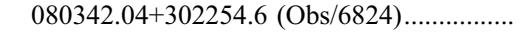 & 2.03 & 2006 Sep 06 & 7 & 4.0 & HS $0800+3031$ & $\mathrm{PC}$ \\
\hline $081331.28+254503.0(\mathrm{Obs} / 3023) \ldots \ldots \ldots \ldots \ldots \ldots$ & 1.51 & 2002 Jan 30 & 3 & 5.0 & HS $0810+2554$ & AC, lensed \\
\hline $084401.95+050357.9(\mathrm{Obs} / 6815) \ldots \ldots \ldots \ldots \ldots \ldots$ & 3.35 & 2006 Feb 18 & 7 & 3.8 & $\ldots$ & PC, BAL, RLQ \\
\hline 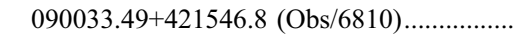 & 3.29 & 2006 Feb 09 & 7 & 3.9 & HS $0857+4227$ & $\mathrm{PC}$ \\
\hline 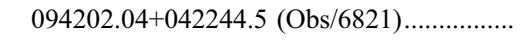 & 3.28 & 2006 Feb 08 & 7 & 4.1 & $\ldots$ & $\mathrm{PC}$ \\
\hline 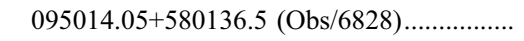 & 3.96 & 2006 Jan 29 & 7 & 3.9 & PSS J0950+5801 & $\mathrm{PC}$ \\
\hline 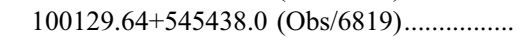 & 1.76 & 2006 Jan 29 & 7 & 4.0 & Mrk 132 & $\mathrm{PC}$ \\
\hline 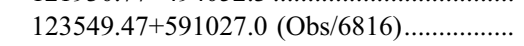 & 2.82 & 2006 Jul 29 & 7 & 3.9 & SBS $1233+594$ & $\mathrm{PC}$ \\
\hline $123641.46+655442.0(\mathrm{Obs} / 6817) \ldots \ldots \ldots \ldots \ldots \ldots$ & 3.39 & 2006 Aug 29 & 7 & 4.0 & $\ldots$ & $\mathrm{PC}$ \\
\hline $135044.67+571642.8(\mathrm{Obs} / 6825) \ldots \ldots \ldots \ldots \ldots \ldots$ & 2.91 & 2006 Oct 15 & 7 & 3.0 & SBS $1348+575$ & PC \\
\hline 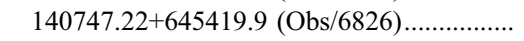 & 3.08 & 2006 Sep 16 & 7 & 3.7 & $\ldots$ & PC \\
\hline $142123.98+463317.8(\mathrm{Obs} / 6823) \ldots \ldots \ldots \ldots \ldots \ldots$ & 3.37 & 2006 Sep 15 & 7 & 3.9 & $\ldots$ & $\mathrm{PC}$ \\
\hline 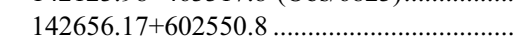 & 3.19 & 1993 Nov 01 & $\ldots$ & $4.1^{\mathrm{c}}$ & SBS $1425+606$ & AR, Reimers et al. (1995) \\
\hline $143835.95+431459.2(\mathrm{Obs} / 6812) \ldots \ldots \ldots \ldots \ldots \ldots$ & 4.61 & 2006 Sep 16 & 7 & 3.7 & $\ldots$ & $\mathrm{PC}$ \\
\hline $144542.75+490248.9$ & 3.88 & 1993 Jul 12 & $\ldots$ & $5.7^{\mathrm{c}}$ & & $\mathrm{AR}$ \\
\hline $152156.48+520238.4(\mathrm{Obs} / 6808) \ldots \ldots \ldots \ldots \ldots \ldots$ & 2.19 & 2006 Jul 16 & 7 & 4.1 & $\ldots$ & $\mathrm{PC}$ \\
\hline $152553.89+513649.1$ & 2.88 & 2001 Dec 08 & $\ldots$ & 24.4 & CSO 755 & AX, BAL, Shemmer et al. (2005a) \\
\hline $161434.67+470420.0(\mathrm{Obs} / 4127) \ldots \ldots \ldots \ldots \ldots \ldots$ & 1.86 & 2003 Dec 20 & 4 & $2.6^{\mathrm{c}}$ & RX J1614.5+4704 & AC, Bade et al. (1995) \\
\hline 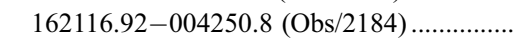 & 3.70 & 2001 Sep 05 & 2 & 1.6 & $\ldots$ & AC, Bechtold et al. (2003) \\
\hline 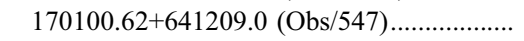 & 2.74 & 2000 Oct 31 & 1 & $39.4^{\mathrm{c}}$ & HS $1700+6416$ & AC, Reimers et al. (1995), Akylas et al. (2006) \\
\hline
\end{tabular}

a The Chandra exposure time has been corrected for detector dead time.

${ }^{\mathrm{b}} \mathrm{PC}=$ pointed Chandra observation; $\mathrm{AC}=$ archival Chandra observation; $\mathrm{AR}=$ archival ROSAT observation; $\mathrm{AX}=$ archival XMM-Newton observation. For sources with archival X-ray data, we list papers where the data were originally published, when possible.

c The mean effective exposure time for the aperture used for sources at large off-axis angles.

observations, and we include these quasars in our analyses below as appropriate. After these two additions, we expect $\lesssim 15 \%$ incompleteness for $M_{i}<-29.28$ broad-line quasars at $z \approx 1.5-4.5$ in the area covered by the SDSS DR 3 quasar catalog. The observation log of our sample, including these two additional sources, appears in Table 1; this 34 object sample will hereafter be referred to as the "core" sample.

In some analyses below, we will complement our core sample with 25 additional comparably luminous $\left(M_{i} \lesssim-29\right)$ quasars at $z \gtrsim 4$ that lie outside the area covered by the SDSS DR3 quasar catalog. The X-ray properties of these quasars have been studied by Vignali et al. $(2003,2005)$ and Shemmer et al. $(2005 \mathrm{~b})$ and appear in Table 2 . These quasars were discovered by the DPOSS and other large-area surveys, and their basic optical properties are consistent with those of our SDSS quasars. This complementary sample is required to improve our statistical coverage at $z>4$, which would otherwise be poor. Since the X-ray and optical properties of these objects were investigated as a whole in S06, this subsample will be referred to as the complementary high-luminosity $z \gtrsim 4$ quasars from S06. We have not added highly luminous quasars outside the SDSS DR3 area at $z<4$ from other surveys, since such quasars generally do not have systematic sensitive X-ray coverage.

\subsubsection{Radio-loud, Broad Absorption Line, and Lensed Quasars}

Radio-loud quasars (RLQs) are known to have jet-linked X-ray emission components that generally lead to higher X-ray-tooptical luminosity ratios than those of radio-quiet quasars (RQQs; e.g., Worrall et al. 1987). Given this finding, it is important to consider RLQs and RQQs separately in statistical analyses of quasar $\mathrm{X}$-ray properties. We quantify radio loudness using the radioloudness parameter $R$, defined as $R=f_{5 \mathrm{GHz}} / f_{4400 \AA}$ (Kellermann et al. 1989). We classify any quasar with $R>10$ as radio-loud; details of our $R$ calculations are given in $\S 3$. Two of the quasars in our 34 object core sample, SDSS J0844+0503 and SDSS J1733+ 5400 , are RLQs. Both objects are only mildly radio-loud $(R \approx 19$ and $R \approx 10$, respectively) and were observed as part of our Chandra Cycle 7 observations. Given the radio coverage of all of our sources (including tight upper limits of $R<0.5-4$ on many of them), we do not expect there to be any RLQs that have not 
TABLE 2

X-Ray Properties of Complementary $z \gtrsim 4$ Sources with $M_{i} \lesssim-29$

\begin{tabular}{|c|c|c|c|c|c|}
\hline Name & $z$ & Total X-Ray Counts & $f_{\mathrm{X}}^{\mathrm{a}}$ & $\alpha_{\mathrm{ox}}$ & Reference \\
\hline PSS $0133+0400(\mathrm{Obs} / 3152) .$. & 4.15 & 36 & 20.8 & -1.64 & 1 \\
\hline 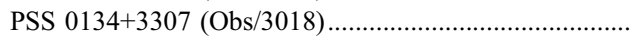 & 4.53 & 16 & 11.5 & -1.68 & 1 \\
\hline 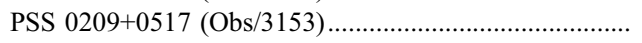 & 4.14 & 22 & 15.2 & -1.75 & 1 \\
\hline 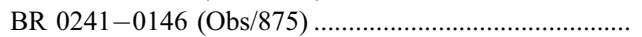 & 4.06 & 12 & 4.3 & -1.87 & 1 \\
\hline 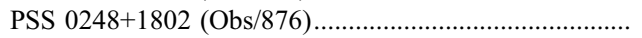 & 4.43 & 14 & 21.2 & -1.64 & 1 \\
\hline 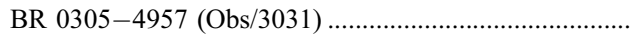 & 4.73 & 3 & 2.1 & -1.94 & 1 \\
\hline 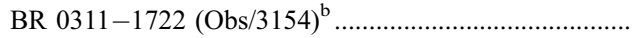 & 4.00 & 7 & 4.1 & -1.93 & 1 \\
\hline 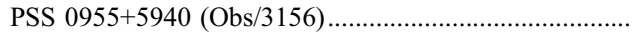 & 4.34 & 10 & 6.0 & -1.81 & 1 \\
\hline 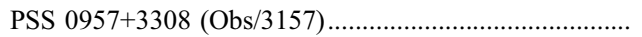 & 4.20 & 17 & 11.3 & -1.74 & 1 \\
\hline 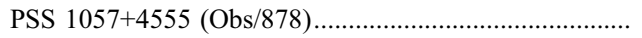 & 4.12 & 24 & 25.3 & -1.70 & 1 \\
\hline 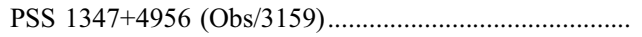 & 4.51 & 30 & 17.5 & -1.78 & 1 \\
\hline 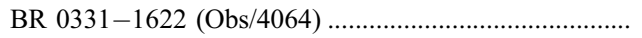 & 4.36 & 14 & 8.5 & -1.86 & 2 \\
\hline 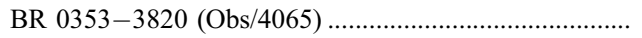 & 4.55 & 55 & 44.7 & -1.54 & 2 \\
\hline 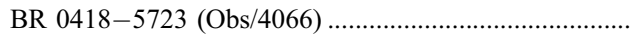 & 4.46 & 7 & 7.0 & -1.87 & 2 \\
\hline 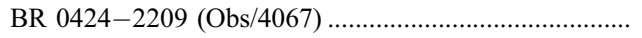 & 4.32 & 11 & 8.6 & -1.81 & 2 \\
\hline 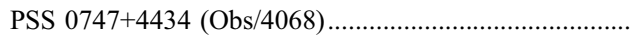 & 4.43 & 5 & 5.7 & -1.81 & 2 \\
\hline PSS $1058+1245$ (Obs/4069) & 4.33 & 5 & 2.4 & -2.07 & 2 \\
\hline 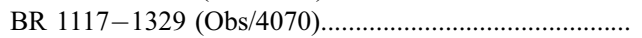 & 3.96 & 2 & 1.8 & -2.03 & 2 \\
\hline 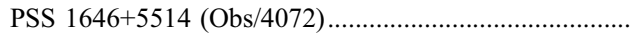 & 4.04 & 5 & 5.0 & -2.00 & 2 \\
\hline 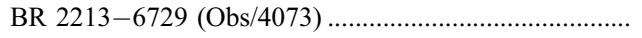 & 4.47 & 20 & 14.9 & -1.64 & 2 \\
\hline 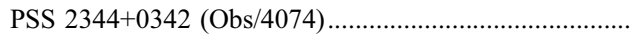 & 4.24 & $\ldots$ & $<2.6$ & $<-1.98$ & 2 \\
\hline Q0000-263 & 4.10 & $1229^{\mathrm{c}}$ & 12.6 & -1.70 & 3 \\
\hline PSS 0926+3055 & 4.19 & $1156^{\mathrm{c}}$ & 39.0 & -1.76 & 3 \\
\hline 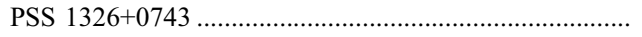 & 4.09 & $963^{\mathrm{c}}$ & 27.9 & -1.76 & 3 \\
\hline 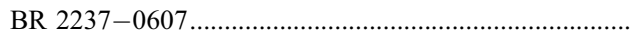 & 4.56 & $306^{\mathrm{c}}$ & 8.5 & -1.74 & 3 \\
\hline
\end{tabular}

NotE.-Quasars are first sorted by reference, then further sorted by right ascension.

${ }^{\text {a }}$ Galactic-absorption-corrected flux in the observed $0.5-2 \mathrm{keV}$ band in units of $10^{-15} \mathrm{ergs} \mathrm{cm}^{-2} \mathrm{~s}^{-1}$.

b This source appears in Vignali et al. (2003) under its older designation BR 0308-1734.

${ }^{c}$ Since these sources were observed by XMM-Newton, we quote the total counts from the pn detector.

References.-(1) Vignali et al. 2003; (2) Vignali et al. 2005; (3) Shemmer et al. 2005b.

been identified as such. Although it is possible that there may still be some jet-linked X-ray contribution for sources with $R<$ 10, we use equation (2) of Worrall et al. (1987) to estimate a limit of $\leqslant 2 \%$ on the fraction of X-ray emission from our RQQs that is jet related.

Owing to intrinsic X-ray absorption, BAL quasars also require special consideration in statistical analyses of quasar X-ray properties (e.g., Gallagher et al. 2006 and references therein). Using a catalog of BAL quasars from DR3 compiled by Trump et al. (2006), we found six potential BAL quasars in our SDSS sample. The three we admit as BAL quasars are SDSS J0844+0503, SDSS $\mathrm{J} 1525+5136$, and SDSS J2313+0034. All three have positive "balnicity" indices (see Trump et al. 2006); note that SDSS J0844+0503 is a mildly radio-loud BAL quasar. The other three potential BAL quasars, SDSS J1001+5454, SDSS J1407+6454, and SDSS J1426+6025, have UV absorption but do not formally satisfy the positive balnicity criterion, so we do not remove them from our analyses below. The removal of these three sources from our main analyses does not significantly affect any of the results. Three of these quasars (SDSS J0844+0503, SDSS J1001+ 5454, and SDSS J1407+6454) were observed as part of our Chandra Cycle 7 observations; the remaining ones have archival X-ray coverage. Given that the redshifts of our quasars are sufficiently high (with the possible exception of SDSS J0813+2545) so that the definitive $\mathrm{C}$ IV BAL transition lies within the spectral range covered by the SDSS spectra (this requires $z \gtrsim 1.5$ ), we do not expect there to be any unidentified BAL quasars within our sample. Note that the complementary high-luminosity $z \gtrsim 4$ quasars from S06 have been chosen to be radio-quiet, non-BAL quasars.
Three quasars with archival X-ray observations described in this paper, SDSS J0145-0945, SDSS J0813+2545, and APM $08279+5255$, are gravitationally lensed. SDSS J0145-0945 and APM $08279+5255$ have flux-amplification factors of $\approx 3$ and $\approx 100$, respectively (e.g., Surdej et al. 1988; Egami et al. 2000; Lehar et al. 2000; E. O. Ofek 2006, private communication), and we have calculated the flux-amplification factor of SDSS J0813+ 2545 to be $\approx 6$ (based on the $V$-magnitudes taken from the discovery paper of Reimers et al. 2002). After correcting for flux amplification due to lensing, none of these quasars satisfies our $M_{i}=-29.28$ cutoff. Therefore, we present the basic X-ray properties of these three quasars below but exclude them from most of our statistical analyses. Since our sample consists of sources at the top of the quasar luminosity function (resulting in a strong magnification bias), the expected fraction of lensed quasars is of the order of a few percent (e.g., Turner et al. 1984; E. O. Ofek 2007 , private communication); this is consistent with the $\sim 10 \%$ fraction of lensed quasars that we find for our highly luminous sample. We do not expect unresolved $\left(\leqslant 1^{\prime \prime}\right)$ gravitational lenses to be affecting our results materially, and we constrain extended $\mathrm{X}$-ray emission in $\S 2.2$.

\subsection{Chandra Observations and Data Reduction}

Our 21 Chandra Cycle 7 targets (see $\S 2.1 .1$ ) were observed using the Advanced CCD Imaging Spectrometer (ACIS; Garmire et al. 2003) with the aim point on the S3 CCD. The requested "snapshot" exposure for each target was 4 ks. All targets were placed near the aim point; with the exceptions of SDSS J1350+ 5716, SDSS J1421+4633, and SDSS J1521+5202, all were strongly detected with $\approx 10-150$ counts from 0.5 to $8 \mathrm{keV}$ (details 
TABLE 3

X-Ray Counts, Band Ratios, and Effective Photon Indices of the Core Sample

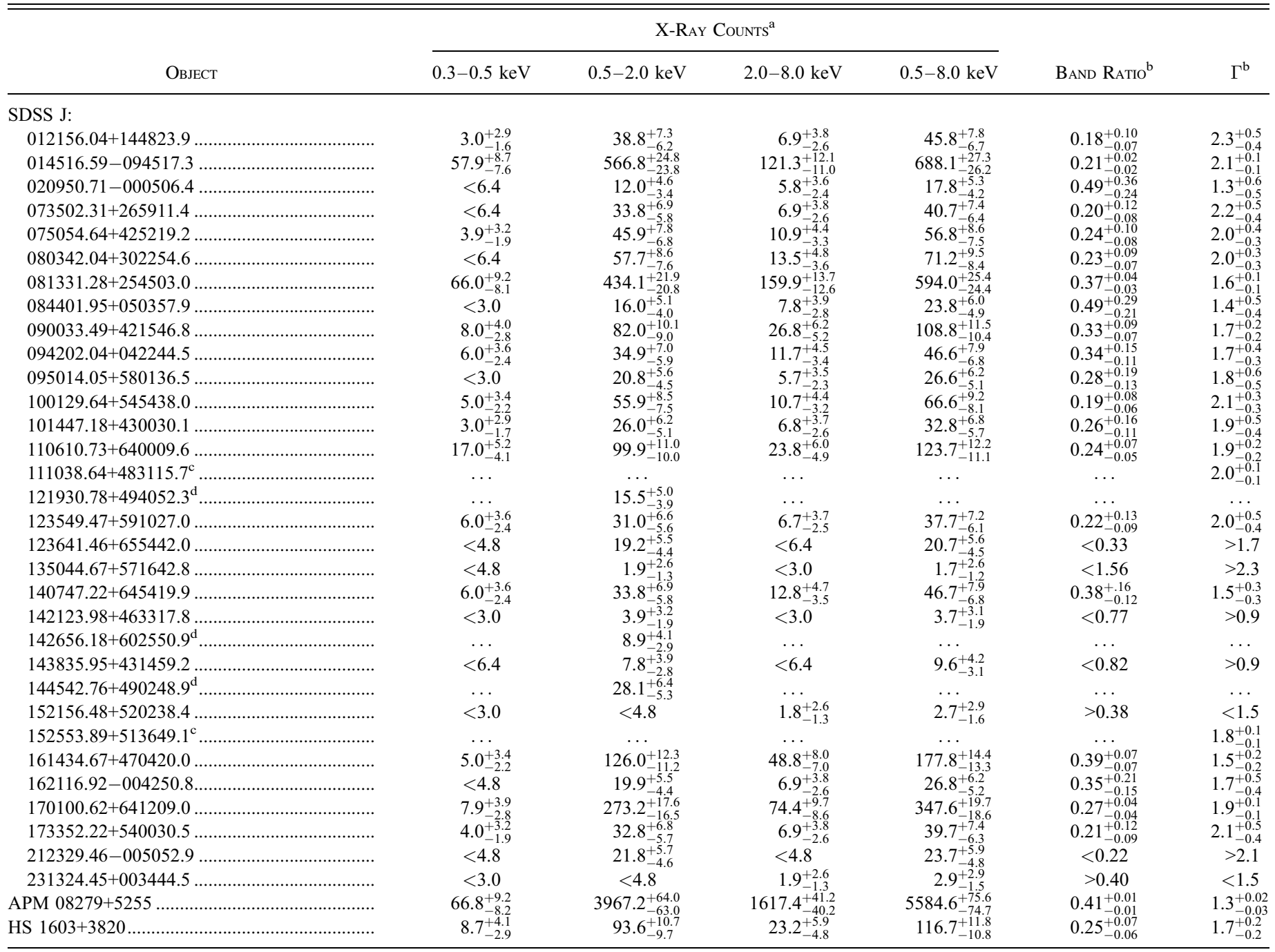

${ }^{a}$ Using Poisson statistics, the errors on the X-ray counts were calculated according to Tables 1 and 2 of Gehrels (1986) and correspond to the $1 \sigma$ level. Upper limits on X-ray counts were computed according to Kraft et al. (1991) and are at the $95 \%$ confidence level. Upper limits of 3.0, 4.8, and 6.4 correspond to finding 0,1 , and 2 X-ray counts within an extraction region of $1^{\prime \prime}$ centered on the optical position of the quasar, considering the background negligible within that region.

b We define the band ratio as the hard-band counts divided by the soft-band counts. Errors for the band ratio and power-law photon index were calculated at the $1 \sigma$ level following the "numerical method" described in $\S 1.7 .3$ of Lyons (1991). When the number of counts is small, this method avoids the failure of the standard approximatevariance formula. The photon indices have been obtained by applying the correction required to account for the quantum-efficiency decay of ACIS at low energies. Note that because the sources in this sample have been observed in different Chandra cycles, the quoted band ratios cannot be directly compared with each other, due to the time dependence of the ACIS quantum efficiency.

${ }^{c}$ The X-ray properties (i.e., $\Gamma$ and the soft-band flux) of SDSS J1110+4831 and SDSS J1525+5136 have been calculated from XMM-Newton observations, which observes in a different energy band than Chandra. Therefore we do not have values to quote for the X-ray counts.

${ }_{\mathrm{d}}$ The X-ray properties of these objects were determined from soft-band count measurements taken from archival ROSAT data. Therefore we do not quote values for the $\mathrm{X}$-ray counts in the other bands.

on SDSS J1521+5202 appear in $\S 4$ ). Faint mode was used for the event telemetry format, and all observations were free from strong background flares.

Data analysis was carried out using standard CIAO $^{5}$ version 3.2 routines, and only events with ASCA grades $0,2,3,4$, and 6 were used. The wavdetect algorithm (Freeman et al. 2002) was used for source detection with wavelet scales of $1, \sqrt{2}, 2,2 \sqrt{2}$, and 4 pixels. We adopted a wavdetect false-positive probability threshold of $10^{-4}$. The probability of spurious detections is very low, given our a priori knowledge of the locations of our sources and the subarcsecond on-axis resolution of Chandra. All Cycle 7 targets were detected by Chandra.
We report in Table 3 the counts detected in the ultrasoft band $(0.3-0.5 \mathrm{keV})$, the soft band $(0.5-2 \mathrm{keV})$, the hard band $(2-8 \mathrm{keV})$, and the full band $(0.5-8 \mathrm{keV})$. Manual aperture photometry with a $3^{\prime \prime}$ radius aperture was used to derive the counts. Also in Table 3 we give the band ratio (the hard-band counts divided by the soft-band counts) and the effective power-law photon index $\Gamma$, assuming an X-ray photon spectrum of the form $N_{E} \propto E^{-\Gamma}$ across the full band. This photon index was calculated from the band ratio using the Chandra PIMMS ${ }^{6}$ version 3.6a tool; we used the Cycle 7 instrument response in PIMMS, which accounts for the time-dependent quantum-efficiency decay of ACIS at low energies (caused by a thin layer of molecular buildup on the ACIS filter). 
We examined the data for the presence of extended X-ray emission (e.g., due to gravitational lensing or jets) by comparing the radial profiles of our sources with their expected, normalized point-spread functions (PSFs). None of the Cycle 7 targets showed extended X-ray emission (but see $\S 2.1 .3$ ). We also did not find extended X-ray emission for any of the non-gravitationally lensed Chandra archival quasars.

We checked for an excess of X-ray companions near our quasars by searching projected circular regions of $\approx 300 \mathrm{kpc}$ in radius centered on each source. We found the number of companions within those fields to be consistent with expectations from the cumulative number counts from X-ray surveys (e.g., Bauer et al. 2004).

Rapid variability within the observations of our sources was searched for by applying Kolmogorov-Smirnov tests to the photon arrival times of quasars with $\gtrsim 10$ counts. No variability was detected, which is not unexpected given the short exposure times of our observations ( $\$ 30$ minutes in the rest frame).

\subsection{Archival X-Ray Data}

Archival data exist and were utilized for 11 of the sources in our core sample; in Table 1 we give references to past X-ray studies when available. The six quasars SDSS J0145-0945 (PI: G. P. Garmire), SDSS J0813+2545 (PI: R. S. Priddey), SDSS J1614+ 4704 (PI: G. Fossati), SDSS J1621-0042 (PI: J. Bechtold), SDSS J1701+6412 (PI: L. P. Van Speybroeck), and SDSS J2313+ 0034 (PI: S. F. Anderson) were observed previously with Chandra. With the exception of SDSS J2313+0034, all of these sources have $>10$ counts and, aside from SDSS J1614+4704 and SDSS J1701+6412, have been observed on-axis. For SDSS J1614+ 4704 and SDSS J1701+6412, we used apertures with radii of $30^{\prime \prime}$ and $8^{\prime \prime}$, respectively, to account for PSF broadening at large offaxis angles. We averaged the exposure maps over the same respective apertures when calculating the count rates. For SDSS J01450945 , which is a gravitationally lensed quasar, we used an aperture with a radius of $4^{\prime \prime}$ in order to enclose the counts from both images. Although SDSS J0813+2545 is also gravitationally lensed, the angular separation between the images is small enough for a $3^{\prime \prime}$ radius aperture to suffice. Data reduction for these sources was carried out in a manner similar to that described in $\S 2.2$, with the proper correction applied to each source for the time-dependent quantum-efficiency decay of ACIS at low energies.

Archival ROSAT data were used for three of the quasars: SDSS J1219+4940 (PI: J. Liebert), SDSS J1426+6025 (PI: D. Reimers), and SDSS J1445+4902 (PI: U. Herbstmeier). These objects were detected by the ROSAT PSPC instrument, with 15.5, 8.9, and 28.1 counts, respectively, in the $0.5-2.0 \mathrm{keV}$ band. SDSS J1219+4940 and SDSS J1445+4902 have off-axis angles of 20.6 and 16.1', while SDSS J1426+6025 was observed on-axis. The counts were derived for these quasars using aperture sizes of $45^{\prime \prime}$ for SDSS $\mathrm{J} 1426+6025,140^{\prime \prime}$ for SDSS J1219+4940, and $100^{\prime \prime}$ for SDSS $\mathrm{J} 1445+4902$; background was estimated by placing circular apertures on regions where no other X-ray sources were present. Using PIMMS to extrapolate our measured $0.5-2.0 \mathrm{keV}$ count rate for SDSS J1426+6025 to the full ROSAT energy range $(\approx 0.1-$ $2.4 \mathrm{keV}$ ), we found our value was consistent with that previously published by Reimers et al. (1995).

The remaining two archival sources in our sample, SDSS J1110+4831 and SDSS J1525+5136, were previously observed with XMM-Newton. SDSS J1110+4831 has its X-ray properties described in detail in Page et al. (2005). We have reanalyzed the $X M M$-Newton data using standard XMM-Newton Science Analysis System (ver. 6.5.0) tasks and found results consistent with those presented in Page et al. (2005); the X-ray values we quote in Tables 3 and 4 are taken from our analysis. Similarly, the X-ray data for SDSS J1525+5136 have appeared in Page et al. (2005), and this quasar has been studied in detail in Shemmer et al. (2005a); we use the data from Shemmer et al. (2005a) in our analysis.

\section{X-RAY, OPTICAL, AND RADIO PROPERTIES OF THE CORE SAMPLE}

In Table 4 we list the main X-ray, optical, and radio properties of our core sample.

Column (1).- The SDSS J2000.0 quasar coordinates, accurate to $\sim 0.1^{\prime \prime}$.

Column (2).-Galactic column density in units of $10^{20} \mathrm{~cm}^{-2}$, calculated using $\mathrm{COLDEN}^{7}$ with the data from Stark et al. (1992).

Column (3).- The monochromatic $\mathrm{AB}$ magnitude at a restframe wavelength of $1450 \AA\left(\mathrm{AB}_{1450}=-2.5 \log f_{1450} \AA-48.6\right.$; Oke \& Gunn 1983). The $\mathrm{AB}_{1450}$ magnitudes were calculated from the spectra after applying corrections due to Galactic extinction and fiber light-loss. The fiber light-loss correction was calculated as the average difference between the synthetic $g, r$, and $i$ magnitudes (i.e., the integrated flux across each respective bandpass in the SDSS spectrum) and the photometric $g, r$, and $i$ magnitudes, assuming no flux variation between the spectroscopic and photometric epochs. The $\mathrm{AB}_{1450}$ magnitudes for the two quasars that were missed by the SDSS selection criteria, APM $08279+5255$ and HS $1603+3820$, were calculated from the photometric $i$ magnitudes.

Column (4).- The absolute $i$-band magnitude, taken from the SDSS DR3 quasar catalog; for APM 08279+5255 and HS $1603+3820$, we calculated this value from the photometric $i$ magnitude, correcting for Galactic extinction.

Columns (5) and (6).- - The flux density and luminosity at a rest-frame wavelength of $2500 \AA$ computed from the magnitude in column (3), assuming a UV-optical power-law slope of $\alpha=$ -0.5 (Vanden Berk et al. 2001), where $F_{\nu} \propto \nu^{\alpha}$.

Columns (7) and (8).-The count rate in the observed-frame $0.5-2.0 \mathrm{keV}$ band and the corresponding flux, corrected for Galactic absorption and the quantum-efficiency decay of Chandra ACIS at low energy. The fluxes have been calculated using PIMMS, assuming a power-law model with $\Gamma=2.0$, which is a typical photon index for luminous AGNs (e.g., Reeves \& Turner 2000; Page et al. 2005; Piconcelli et al. 2005; Shemmer et al. 2005b; Vignali et al. 2005; see $\S 5.1$ for direct justification).

Columns (9) and (10).-The flux density and luminosity at a rest-frame energy of $2 \mathrm{keV}$, computed assuming $\Gamma=2.0$ and corrected for the quantum-efficiency decay of Chandra ACIS at low energy.

Column (11).- The luminosity in the rest-frame $2-10 \mathrm{keV}$ band.

Column (12).-The X-ray-to-optical power-law slope, $\alpha_{\mathrm{ox}}$, defined as

$$
\left.\alpha_{\mathrm{ox}} \equiv \frac{\log \left(f_{2 \mathrm{keV}} / f_{2500 \AA}\right)}{\log \left(\nu_{2 \mathrm{keV}} / \nu_{2500} \AA\right.}\right)=0.3838 \log \left(f_{2 \mathrm{keV}} / f_{2500 \AA}\right),
$$

where $f_{2 \mathrm{keV}}$ and $f_{2500 \AA}$ are the monochromatic flux densities at rest-frame $2 \mathrm{keV}$ and $2500 \AA$, respectively.

Column (13).- The difference between the measured $\alpha_{\text {ox }}$ (from col. [12]) and the predicted $\alpha_{\text {ox }}$ (quoted as $\Delta \alpha_{\text {ox }}$ ), given the UV luminosity from column (6), based on the established $\alpha_{\mathrm{ox}}-L_{2500 \AA}$ relation (given as eq. [2] of S06). The statistical significance of this difference is also given in units of $\sigma$, where $\sigma=0.146$ for $31<\log \left(L_{2500} \AA\right)<32$, and $\sigma=0.131$ for $32<$ $\log \left(L_{2500 \AA}\right)<33$ (see Table 5 of S06).

Column (14).-The radio-loudness parameter $R$ (see $\S 2.1 .3$ ). The $f_{4400} \AA$ values were calculated by extrapolating from the

\footnotetext{
${ }^{7}$ See http://cxc.harvard.edu/toolkit/colden.jsp.
} 
TABLE 4

X-Ray, Optical, and Radio Properties of the Core Sample

\begin{tabular}{|c|c|c|c|c|c|c|c|c|c|c|c|c|c|}
\hline $\begin{array}{l}\text { Object } \\
\text { (1) }\end{array}$ & $\begin{array}{l}N_{\mathrm{H}} \\
(2)\end{array}$ & $\begin{array}{c}\mathrm{AB}_{1450} \\
\text { (3) }\end{array}$ & $\begin{array}{l}M_{i} \\
(4)\end{array}$ & $\begin{array}{c}f_{2500 \AA^{\mathrm{a}}} \\
\text { (5) }\end{array}$ & $\begin{array}{c}\log \left(L_{\nu}\right) \\
2500 \AA \\
(6)\end{array}$ & $\begin{array}{c}\text { Count Rate } \\
\text { (7) }\end{array}$ & $\begin{array}{l}f_{\mathrm{X}}{ }^{\mathrm{c}} \\
(8)\end{array}$ & $\begin{array}{l}f_{2 \mathrm{keV}}{ }^{\mathrm{d}} \\
(9)\end{array}$ & $\begin{array}{c}\log \left(\nu L_{\nu}\right) \\
2 \mathrm{keV} \\
(10)\end{array}$ & $\begin{array}{c}\log L \\
2-10 \mathrm{keV} \\
(11)\end{array}$ & $\begin{array}{l}\alpha_{\text {ox }} \\
(12)\end{array}$ & $\begin{array}{c}\Delta \alpha_{\mathrm{ox}}(\sigma)^{\mathrm{e}} \\
(13)\end{array}$ & $\begin{array}{c}R \\
(14)\end{array}$ \\
\hline \multicolumn{14}{|l|}{ SDSS J: } \\
\hline $012156.04+144823.9$ & 3.92 & 17.0 & -29.29 & 6.83 & 32.09 & $9.9_{-1.6}^{+1.9}$ & $41.2_{-6.6}^{+7.7}$ & $23.8_{-3.8}^{+4.5}$ & $45.31_{-0.08}^{+0.07}$ & 45.52 & -1.71 & $+0.05(0.36)$ & $<2.9^{\mathrm{f}}$ \\
\hline $014516.59-094517.3 \ldots \ldots \ldots \ldots \ldots \ldots \ldots \ldots \ldots$ & 2.70 & 16.7 & -29.50 & 9.08 & 32.18 & $42.5_{-1.8}^{+2.0}$ & $189.4_{-7.9}^{+8.4}$ & $105.5_{-4.4}^{+4.7}$ & $45.93_{-0.02}^{+0.02}$ & 46.13 & -1.51 & $+0.20(1.34)^{\mathrm{g}}$ & $<0.4$ \\
\hline $020950.71-000506.4$ & 2.42 & 16.9 & -29.40 & 7.44 & 32.12 & $5.5_{-16}^{+2.1}$ & $21.9_{-62}^{+8.4}$ & $12.6_{-36}^{+4.8}$ & $45.03_{-015}^{+0.14}$ & 45.24 & -1.83 & $-0.07(0.52)$ & $<0.5$ \\
\hline $073502.31+265911.4 \ldots \ldots \ldots \ldots \ldots \ldots \ldots \ldots$ & 5.67 & 16.5 & -29.28 & 11.85 & 32.05 & $8.5_{-1.5}^{+1.0}$ & $37.0_{-6.3}^{+0.5}$ & $16.4_{-2.8}^{+3.0}$ & $44.87_{-0.08}^{+0.08}$ & 45.08 & -1.87 & $-0.11(0.86)$ & 1.0 \\
\hline 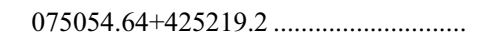 & 4.95 & 16.0 & -29.50 & 17.08 & 32.17 & $11.4_{-1.7}^{+1.9}$ & $48.5_{-7.1}^{+8.3}$ & $21.0_{-3.1}^{+3.6}$ & $44.95_{-0.07}^{+0.07}$ & 45.16 & -1.88 & $-0.11(0.87)$ & $<0.2$ \\
\hline 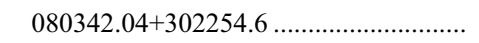 & 4.55 & 16.3 & -29.33 & 12.68 & 32.10 & $14.4_{-1.9}^{+2.2}$ & $60.7_{-8.0}^{+9.1}$ & $27.5_{-3.6}^{+4.1}$ & $45.12_{-0.06}^{+0.06}$ & 45.32 & -1.79 & $-0.03(0.23)$ & $<0.3$ \\
\hline $081331.28+254503.0 \ldots \ldots \ldots \ldots \ldots \ldots \ldots \ldots \ldots$ & 3.80 & 16.2 & -29.40 & 13.45 & 31.89 & $88.7_{-43}^{+4.5}$ & $281.3_{-135}^{+14.2}$ & $105.5_{-51}^{+5.3}$ & $45.47_{-0.02}^{+0.02}$ & 45.68 & -1.58 & $+0.06(0.39)^{\mathrm{g}}$ & $<0.2$ \\
\hline $084401.95+050357.9$ & 3.65 & 17.7 & -29.49 & 3.46 & 31.90 & $4.2_{-11}^{+1.3}$ & $17.3_{-43}^{+5.5}$ & $11.2_{-2.8}^{+3.6}$ & $45.10_{-012}^{+0.12}$ & 45.31 & -1.72 & $+0.01(0.07)$ & 18.9 \\
\hline $090033.49+421546.8 \ldots \ldots \ldots \ldots \ldots \ldots \ldots \ldots \ldots$ & 2.03 & 16.6 & -29.86 & 9.17 & 32.31 & $21.0_{-2.3}^{+2.6}$ & $82.5_{-9.1}^{+10.2}$ & $52.8_{-5.8}^{+6.5}$ & $45.76_{-0.05}^{+0.05}$ & 45.97 & -1.63 & $+0.16(1.24)$ & 1.6 \\
\hline $094202.04+042244.5 \ldots \ldots \ldots \ldots \ldots \ldots \ldots \ldots$ & 3.51 & 17.1 & -29.39 & 6.18 & 32.14 & $8.6_{-1.5}^{+1.7}$ & $35.1_{-5.9}^{+7.1}$ & $22.4_{-3.8}^{+4.5}$ & $45.38_{-0.08}^{+0.08}$ & 45.59 & -1.70 & $+0.06(0.47)$ & $<0.6$ \\
\hline $095014.05+580136.5 \ldots \ldots \ldots \ldots \ldots \ldots \ldots \ldots$ & 1.35 & 17.6 & -29.28 & 3.59 & 32.04 & $5.3_{-1.2}^{+1.4}$ & $20.6_{-4.4}^{+5.5}$ & $15.2_{-3.3}^{+4.1}$ & $45.35_{-0.11}^{+0.10}$ & 45.55 & -1.68 & $+0.07(0.56)$ & $<1.3$ \\
\hline $100129.64+545438.0$ & 0.84 & 15.9 & -29.41 & 17.06 & 32.11 & $14.0_{-19}^{+2.1}$ & $52.9_{-71}^{+8.1}$ & $21.8_{-29}^{+3.3}$ & $44.91_{-0.06}^{+0.06}$ & 45.11 & -1.88 & $-0.12(0.89)$ & $<0.2$ \\
\hline 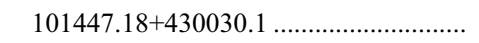 & 1.16 & 16.5 & -30.02 & 11.76 & 32.38 & $6.3_{-1.2}^{+1.5}$ & $24.1_{-4.7}^{+5.1}$ & $14.9_{-2.9}^{+3.5}$ & $45.17_{-0.10}^{+0.09}$ & 45.38 & -1.88 & $-0.08(0.62)$ & $<0.3$ \\
\hline $110610.73+640009.6 \ldots \ldots \ldots \ldots \ldots \ldots \ldots \ldots \ldots . .$. & 1.11 & 16.1 & -29.65 & 14.83 & 32.23 & $27.4_{-2.7}^{+3.0}$ & $104.7_{-10.4}^{+11.6}$ & $50.0_{-5.0}^{+5.96}$ & $45.44_{-0.05}^{+0.05}$ & 45.65 & -1.72 & $+0.06(0.47)$ & $<0.2$ \\
\hline 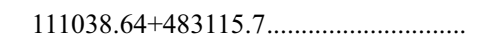 & 1.37 & 16.7 & -29.76 & 9.11 & 32.23 & & $24.0_{-0.4}^{+0.4}$ & $13.7_{-0.2}^{+0.0}$ & $45.10_{-0.01}^{+0.03}$ & 45.30 & -1.85 & $-0.07(0.56)$ & $<0.4$ \\
\hline $121930.78+494052.3 \ldots \ldots \ldots \ldots \ldots \ldots \ldots \ldots$ & 1.83 & 17.0 & -29.29 & 7.27 & 32.07 & $7.2_{-1.8}^{+2.3}$ & $87.8_{-22.1}^{+28.4}$ & $48.5_{-12.2}^{+15.7}$ & $45.58_{-0.13}^{+0.11}$ & 45.79 & -1.60 & $+0.15(1.17)$ & 2.4 \\
\hline $123549.47+591027.0 \ldots \ldots \ldots \ldots \ldots \ldots \ldots \ldots \ldots$ & 1.18 & 16.9 & -29.46 & 7.18 & 32.10 & $8.0_{-1.1}^{+1.9}$ & $30.4_{-5}^{+6.5}$ & $17.4_{-3.1}^{+3.7^{2}}$ & $45.17_{-0.09}^{+0.08}$ & 45.37 & -1.77 & $-0.01(0.10)$ & $<0.5$ \\
\hline $123641.46+655442.0 \ldots \ldots \ldots \ldots \ldots \ldots \ldots \ldots \ldots$ & 1.96 & 17.2 & -29.43 & 6.03 & 32.15 & $4.8_{-1.1}^{+1.4}$ & $18.8_{-4.3}^{+5.4}$ & $12.3_{-2.8}^{+3.5}$ & $45.15_{-0.11}^{+0.11}$ & 45.35 & -1.80 & $-0.03(0.25)$ & $<3.7^{\mathrm{f}}$ \\
\hline 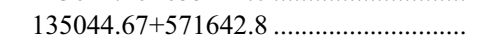 & 1.22 & 17.2 & -29.31 & 5.45 & 32.00 & $0.6_{-0.4}^{-1.9}$ & $2.5_{-1.7}^{+3.4}$ & $1.4_{-1.0}^{+2.0}$ & $44.10_{-0.48}^{+0.37}$ & 44.31 & -2.14 & $-0.40(3.02)$ & $<0.7$ \\
\hline $140747.22+645419.9 \ldots \ldots \ldots \ldots \ldots \ldots \ldots \ldots \ldots . .$. & 1.90 & 17.2 & -29.29 & 6.16 & 32.09 & $11.0_{-1.9}^{+2.24}$ & $43.0_{-7.4}^{+8.7}$ & $26.2_{-4.5}^{+5.0}$ & $45.41_{-0.08}^{+0.08}$ & 45.61 & -1.68 & $+0.08(0.62)$ & $<3.4^{\mathrm{f}}$ \\
\hline $142123.98+463317.8 \ldots \ldots \ldots \ldots \ldots \ldots \ldots \ldots$ & 1.40 & 17.3 & -29.34 & 5.12 & 32.08 & $1.0_{-05}^{+0.8}$ & $3.8_{-19}^{+3.1}$ & $2.5_{-12}^{+2.0}$ & $44.45_{-0.29}^{+0.26}$ & 44.66 & -2.04 & $-0.28(2.15)$ & $<0.7$ \\
\hline $142656.18+602550.9 \ldots \ldots \ldots \ldots \ldots \ldots \ldots \ldots \ldots$ & 1.75 & 16.3 & -30.22 & 13.05 & 32.45 & $2.2_{-0.7}^{+1.0}$ & $26.5_{-8.8}^{+12.2}$ & $16.6_{-5.5}^{+7.7}$ & $45.23_{-0.17}^{+0.17}$ & 45.44 & -1.88 & $-0.07(0.55)$ & $<0.3$ \\
\hline $143835.95+431459.2 \ldots \ldots \ldots \ldots \ldots \ldots \ldots \ldots \ldots$ & 1.61 & 17.6 & -29.60 & 4.00 & 32.18 & $2.1_{-0.7}^{+1.1}$ & $8.2_{-2.9}^{+4.8}$ & $6.9_{-2.4}^{+3.5}$ & $45.11_{-0.19}^{+0.18}$ & 45.31 & -1.83 & $-0.06(0.44)$ & $<1.1$ \\
\hline $144542.76+490248.9 \ldots \ldots \ldots \ldots \ldots \ldots \ldots \ldots$ & 2.27 & 17.4 & -29.41 & 4.06 & 32.07 & $4.9_{-0.9}^{+1.11}$ & $61.1_{-11.5}^{+13.9}$ & $44.5_{-8.4}^{+10.4}$ & $45.80_{-0.09}^{+0.09}$ & 46.01 & -1.52 & $+0.24(1.80)$ & 7.6 \\
\hline $152156.48+520238.4 \ldots \ldots \ldots \ldots \ldots \ldots \ldots \ldots \ldots . .$. & 1.59 & 15.8 & -30.17 & 21.34 & 32.38 & $0.7_{-0.4}^{+0.7}$ & $2.1_{-1.2}^{+2.2}$ & $1.0_{-0.6}^{+0.4}$ & $43.74_{-0.38}^{+0.32}$ & 43.95 & -2.44 & $-0.63(4.82)$ & $<0.1$ \\
\hline $152553.89+513649.1 \ldots \ldots \ldots \ldots \ldots \ldots \ldots \ldots \ldots$ & 1.60 & 16.9 & -29.64 & 7.41 & 32.13 & -0.4 & $129.2_{-13.8}^{+12.2}$ & $74.9_{-8.0}^{-0.0}$ & $45.82_{-0.05}^{+0.03}$ & 45.92 & -1.51 & $+0.25(1.93)$ & $<0.5$ \\
\hline $161434.67+470420.0 \ldots \ldots \ldots \ldots \ldots \ldots \ldots \ldots \ldots$ & 1.20 & 16.4 & -29.36 & 9.59 & 31.91 & $49.1_{-4.4}^{+4.8}$ & $153.3_{-13.7}^{+14.9}$ & $65.5_{-5.8}^{+6.4}$ & $45.43_{-0.04}^{+0.04}$ & 45.63 & -1.60 & $+0.13(0.92)$ & 4.8 \\
\hline 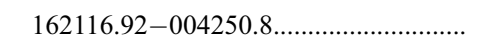 & 7.11 & 17.0 & -29.69 & 5.53 & 32.18 & $12.7_{-2.8}^{+3.5}$ & $44.8_{-9.9}^{+12.4}$ & $31.4_{-7.0}^{+8.7}$ & $45.62_{-0.11}^{+0.11}$ & 45.82 & -1.63 & $+0.14(1.08)$ & $<4.3^{\mathrm{f}}$ \\
\hline $170100.62+641209.0 \ldots \ldots \ldots \ldots \ldots \ldots \ldots \ldots$ & 2.53 & 16.0 & -30.24 & 17.84 & 32.47 & $6.9_{-0.4}^{+0.5}$ & $34.4_{-2.1}^{+2.2}$ & $19.2_{-1.2}^{+1.2}$ & $45.19_{-0.03}^{+0.03}$ & 45.40 & -1.91 & $-0.10(0.73)$ & $<1.1^{\mathrm{f}}$ \\
\hline $173352.22+540030.5 \ldots \ldots \ldots \ldots \ldots \ldots \ldots \ldots \ldots$ & 3.36 & 17.0 & -29.54 & 6.92 & 32.22 & $8.9_{-15}^{+1.8}$ & $36.1_{-63}^{+7.5}$ & $23.9_{-41}^{+5.0}$ & $45.44_{-0.08}^{+0.08}$ & 45.56 & -1.71 & $+0.06(0.48)$ & 10.0 \\
\hline $212329.46-005052.9$ & 4.78 & 16.5 & -29.38 & 11.21 & 32.13 & $5.6_{-1.2}^{+1.5}$ & $23.7_{-5.0}^{+0.32}$ & $11.6_{-2.4}^{+3.0}$ & $44.82_{-0.10}^{+0.10}$ & 45.20 & -1.91 & $-0.15(1.15)$ & $<0.3$ \\
\hline $231324.45+003444.5 \ldots \ldots \ldots \ldots \ldots \ldots \ldots \ldots$ & 4.03 & 16.4 & -29.56 & 13.12 & 32.13 & $2.6_{-1.4}^{+2.6}$ & $11.4_{-6.4}^{+11.4}$ & $5.2_{-2.9}^{+5.4}$ & $44.42_{-0.36}^{+0.10}$ & 44.62 & -2.07 & $-0.31(2.35)$ & $<0.2$ \\
\hline APM $08279+5255$ & 4.05 & 15.1 & -32.00 & 43.87 & 33.11 & $44.7_{-0.7}^{+0.4}$ & $142.9_{-2.2}^{+2.4}$ & $103.9_{-1.6}^{+1.7}$ & $46.17_{-0.01}^{+0.00}$ & 46.37 & -1.78 & $-0.16(1.07)^{\mathrm{g}}$ & $<0.2$ \\
\hline HS $1603+3820 \ldots \ldots$ & 1.32 & 16.1 & -30.05 & 17.94 & 32.41 & $11.3^{+1.3}$ & $35.4^{+4.0}$ & $18.5^{+2.1}$ & $45.11_{-0.05}^{+0.05}$ & 45.23 & -1.91 & $-0.11(0.85)$ & $<0.2$ \\
\hline
\end{tabular}

a Flux density at rest-frame wavelength $2500 \AA \AA$ in units of $10^{-27} \mathrm{ergs} \mathrm{cm}^{-2} \mathrm{~s}^{-1} \mathrm{~Hz}^{-1}$

b Observed count rate computed in the $0.5-2 \mathrm{keV}$ band in units of $10^{-3}$ counts s${ }^{-1}$. The count rates for off-axis sources have been corrected for vignetting.

${ }^{c}$ Galactic-absorption-corrected flux in the observed $0.5-2 \mathrm{keV}$ band in units of $10^{-15} \mathrm{ergs} \mathrm{cm}^{-2} \mathrm{~s}^{-1}$.

d Flux density at rest-frame $2 \mathrm{keV}$ in units of $10^{-32} \mathrm{ergs} \mathrm{cm}^{-2} \mathrm{~s}^{-1} \mathrm{~Hz}^{-1}$.

e The difference between measured and predicted $\alpha_{\mathrm{ox}}\left(\Delta \alpha_{\mathrm{ox}}\right)$, and the significance of that difference $(\sigma)$, based on the S06 $\alpha_{\mathrm{ox}}-L_{\nu}(2500 \AA)$ relation.

${ }^{\mathrm{f}}$ Flux density at an observed-frame frequency of $1.4 \mathrm{GHz}$ taken from the NVSS survey. All other $1.4 \mathrm{GHz}$ flux densities are from the FIRST survey.

${ }^{g}$ The $\Delta \alpha_{\text {ox }}$ values for gravitationally lensed objects have been calculated using lensing-corrected luminosities. 

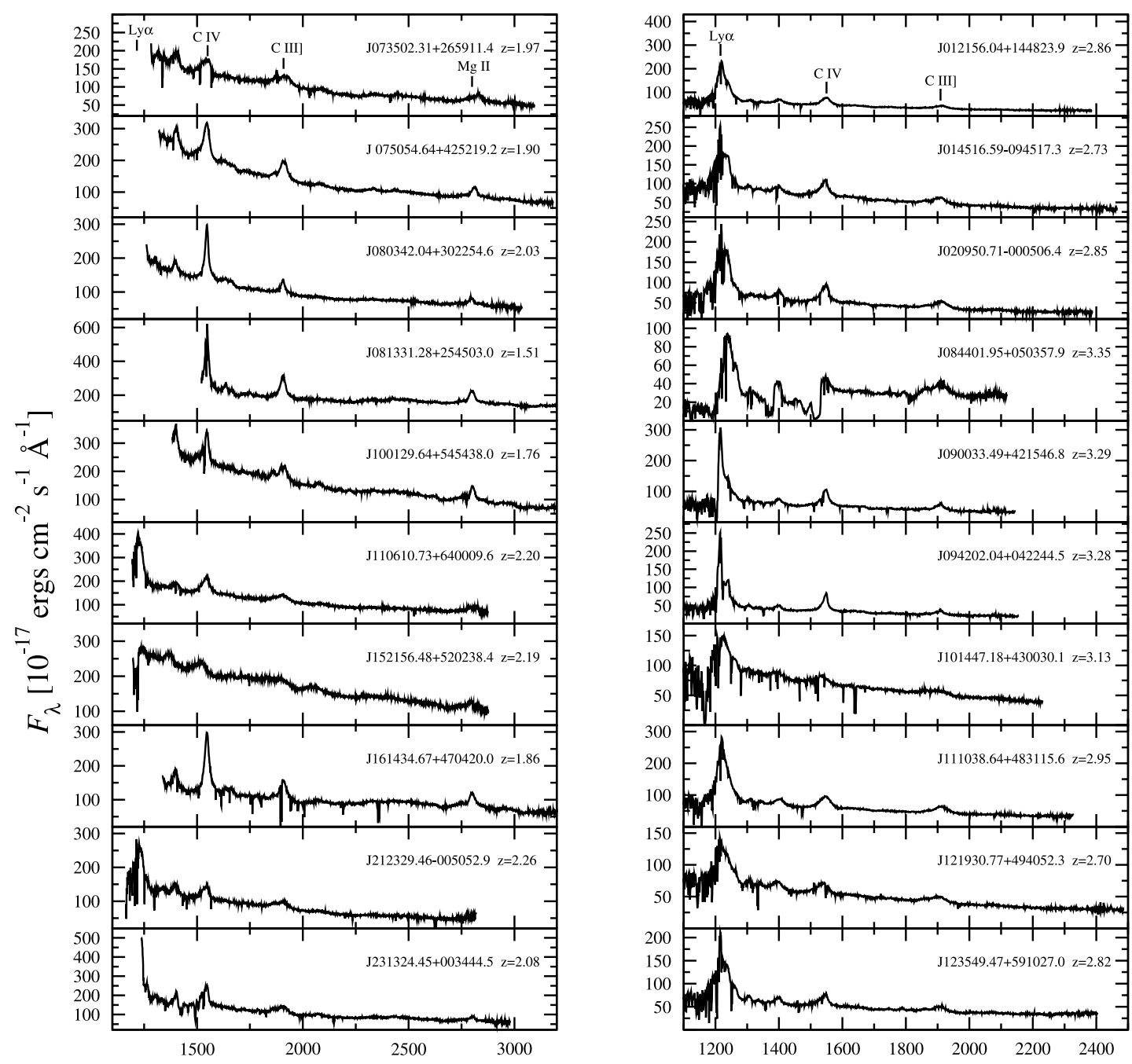

Rest-Frame Wavelength [̊̊]

FIG. 2.-SDSS spectra for our sample of 32 SDSS quasars. Prominent emission lines are marked in the top panel of each column. The spectral resolution is $\approx 2000$. The four columns have been sorted into redshift bins in the following way: the first contains sources from $z \sim 1.5$ to 2.5 , the second and third columns from $z \sim 2.5$ to 3.5 , and the fourth column from $z \sim 3.5$ to 4.5 . Within each column the spectra are sorted by right ascension. SDSS J0844+0503, SDSS J1525+5136, and SDSS J2313+0034 are BAL quasars. Note the C Iv absorption for SDSS J0844+0503, which is also mildly radio-loud, and the interesting spectrum for SDSS J1521+5202, which is shown in greater detail in Fig. 4.

magnitudes in column (3), assuming a UV-optical power-law slope of $\alpha=-0.5$. The $f_{5 \mathrm{GHz}}$ values were calculated using data from the FIRST (Faint Images of the Radio Sky at Twenty cm; Becker et al. 1995) and NVSS (NRAO VLA Sky Survey; Condon et al. 1998) catalogs. The flux density at a rest-frame frequency of $5 \mathrm{GHz}$ was calculated from the flux density at an observed-frame frequency of $1.4 \mathrm{GHz}$ assuming a radio power-law slope of $\alpha=$ -0.8 . Seven of our sources have FIRST radio detections; two of them are RLQs. Upper limits were placed on 20 of our sources at the $3 \sigma$ level, given our a priori knowledge of the positions of all of our sources. Upper limits on the five sources not covered by the FIRST survey were placed using the upper limit on NVSS detection $(\approx 2.5 \mathrm{mJy})$.

For the luminosity values quoted in columns (4), (6), (10), and (11), no lensing corrections have been made (i.e., the fluxes have not been deamplified when determining these values).

\section{OPTICAL SPECTRA AND NOTES ON INDIVIDUAL OBJECTS}

In Figure 2 we present the optical spectra of the 32 SDSS quasars in our sample. Below we comment on sources with particularly interesting optical and/or X-ray properties.
SDSS J014516.59-094517.3 $(z=2.73)$.- This object was first reported as a gravitationally lensed system by Surdej et al. $(1987,1988)$, and it consists of two images separated by $\Delta \theta \approx$ $2^{\prime \prime}$. The optical flux ratio between the two images was found to be $\approx 7$ (Kassiola \& Kovner 1992). Using the Chandra observations taken in 2004 we have calculated an X-ray flux ratio of 12. $4_{-1.7}^{+2.0}$ in the full band (see Fig. 3); this discrepancy between flux ratios is not wholly unexpected, given (1) that the amplitude of flux variations in the X-ray band is typically greater than that in the optical, (2) the likelihood of intrinsic variability of the quasar between the different epochs, and (3) possible microlensing. The optical positions measured from SDSS astrometry agree with the positions of the X-ray centroids of both components to within the expected astrometric accuracy of Chandra $\left(\approx 0.5^{\prime \prime}\right)$. We found $\alpha_{\mathrm{ox}}=-1.51$ for this quasar, which differs from the predicted value from S06 (using a lensing-corrected luminosity) by $1.3 \sigma$.

SDSS J081331.28+254503.0 $(z=1.51)$. - This object is a gravitationally lensed system consisting of four images discovered by Reimers et al. (2002). The angular separation between the two brightest images is $\Delta \theta=0.25^{\prime \prime}$, too small to be resolved by the angular resolution of Chandra $\left(\approx 0.5^{\prime \prime}\right)$. We measure $\alpha_{\text {ox }}=-1.58$ 

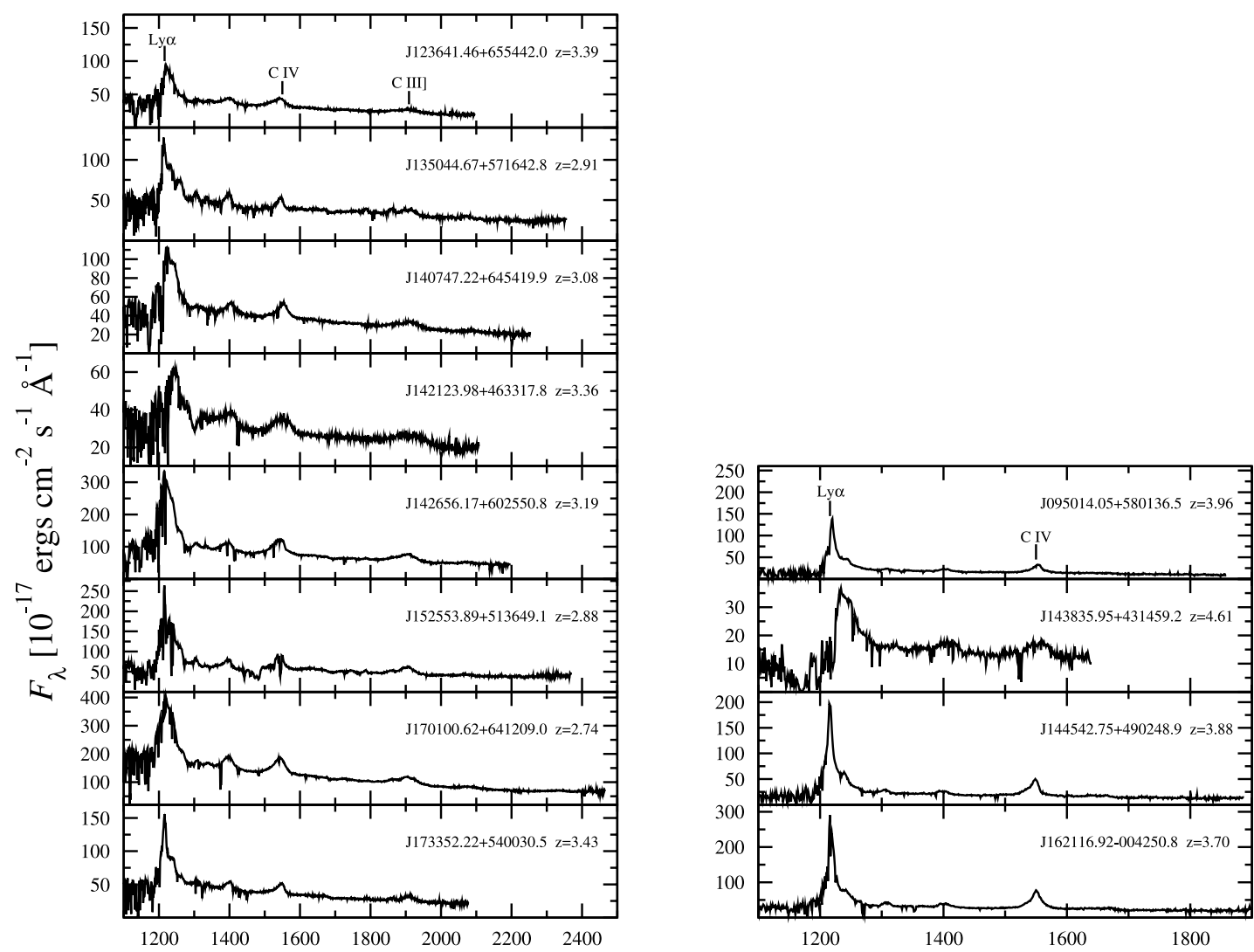

Rest-Frame Wavelength $[\AA]$

FIg. 2-Continued

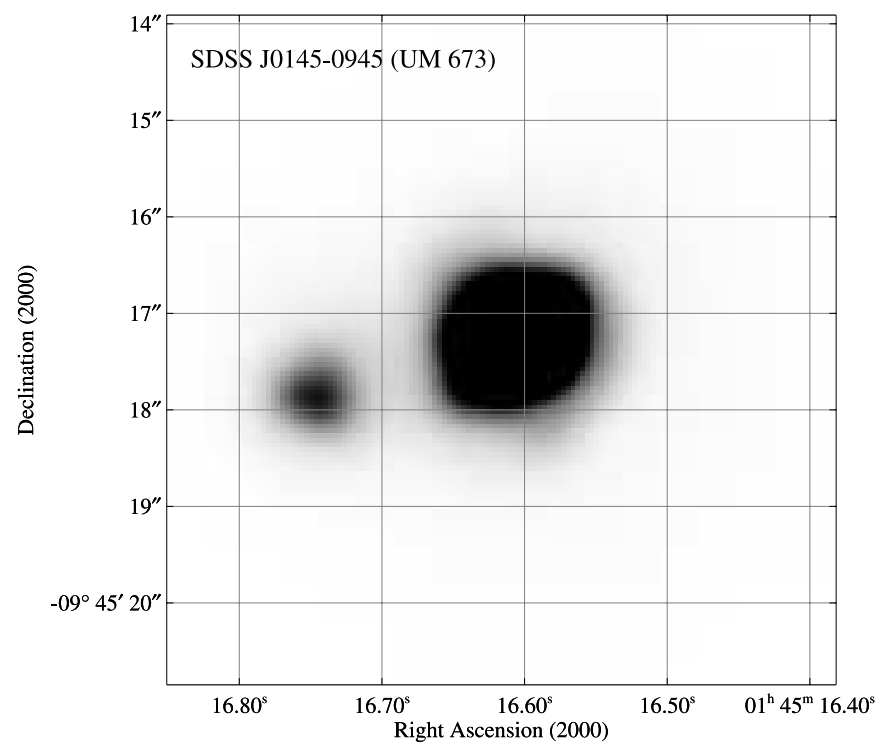

Fig. 3.-Chandra $0.5-8 \mathrm{keV}$ image of the gravitationally lensed quasar SDSS J0145-0945. The image spans $\approx 7^{\prime \prime} \times 7^{\prime \prime}$ on the sky; north is up, and east is to the left. The image has been adaptively smoothed at the $2 \sigma$ level. The two quasar images are separated by $\Delta \theta \approx 2^{\prime \prime}$, and the optical and X-ray flux ratios between them are $\approx 7$ and $\approx 12$, respectively (note that the optical and X-ray flux measurements were taken at different epochs). The positions of the two X-ray images agree with those of the optical images (based on SDSS astrometry) to within the expected Chandra positional error. for this quasar, which differs from the predicted value of S06 (using a lensing-corrected luminosity) by $0.4 \sigma$.

SDSS J084401.95+050357.9 $(z=3.35)$. - This is a BAL RLQ, with a relatively mild radio-loudness parameter of $R=18.9$ and a rest-frame equivalent width $(\mathrm{EW})$ of $\approx 30 \AA$ for the $\mathrm{C}$ IV absorption trough. While the additional jet-linked X-ray emission generally increases the X-ray-to-optical flux ratio, the intrinsic absorption present in BAL quasars tends to reduce that quantity; it is possibly because of these competing effects that we measure $\alpha_{\mathrm{ox}}=-1.72$ for this source, which differs from the predicted value for a non-BAL RQQ with $\log \left(L_{2500 \AA}\right)=31.90$ at a level of only $0.07 \sigma$.

SDSS J135044.67+571642.8 $(z=2.91)$.- - This quasar is somewhat X-ray weak, with $\alpha_{\mathrm{ox}}=-2.14$; this value differs from the predicted value by $3.02 \sigma$. The SDSS spectrum of this object appears in Figure 2, and it shows no obvious UV absorption.

SDSS J152156.48+520238.4 $\left(z=2.19^{8}\right)$.- This exceptionally luminous quasar is the third most optically luminous object in S05. Manual aperture photometry for this quasar measured only 3 counts: 2 in the hard band and 1 in the soft band. X-ray fluxes and other properties were calculated from the full-band count rate using PIMMS since there were not enough counts in the soft band for a detection. This quasar is anomalously X-ray weak, with $\log \left(\nu L_{\nu}\right)_{2 \mathrm{keV}}=43.74$ and a steep $\alpha_{\text {ox }}=-2.44_{-0.15}^{+0.12}$, which is inconsistent with the predicted value at a level of $\approx 5 \sigma$. The SDSS spectrum of this quasar appears in Figure 4. The Ly $\alpha$ line is completely absorbed by several narrow absorption line (NAL) systems, and the high-ionization emission lines are blueshifted

${ }^{8}$ Although the SDSS quotes a redshift of 2.21 , on examination of the spectrum we have measured a redshift of 2.19 based on the $\mathrm{Mg}$ II $\lambda 2798$ line; it is this latter value which we adopt throughout this paper. 


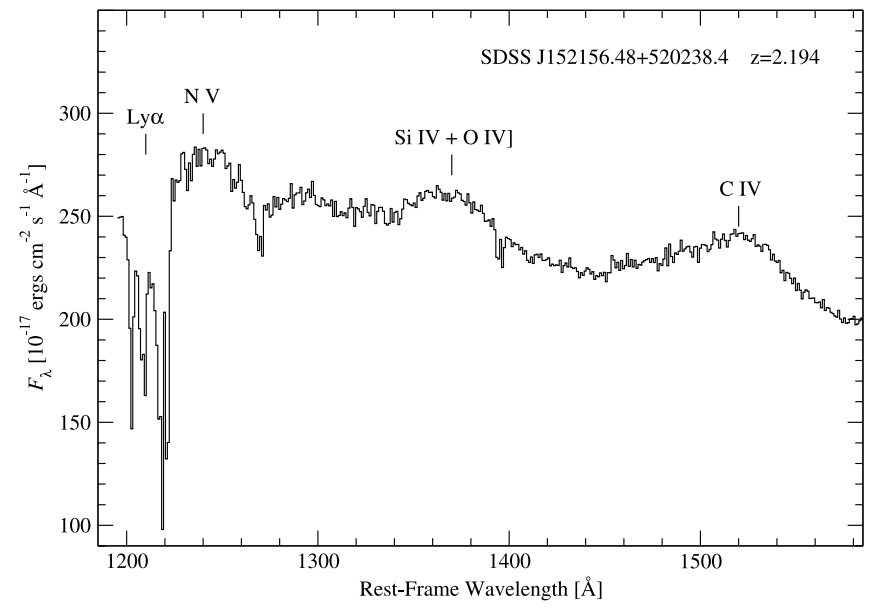

FIG. 4.- SDSS spectrum of SDSS J1521+5202 (see $\S 4$ ). This object shows strong Ly $\alpha$ absorption, which suggests that absorbing material along the line-ofsight is responsible for its anomalous X-ray weakness $\left(\alpha_{\text {ox }}=-2.44\right)$. The spectral resolution is $\approx 2000$.

relative to the quasar's redshift, even when allowing for the revised redshift in footnote 8 . The strong observed UV absorption and the hint of a hard X-ray spectral shape suggest that absorbing material along the line of sight is likely responsible for the X-ray weakness of this quasar (e.g., Brandt et al. 2000; Gallagher et al. 2001). Therefore, we will exclude this quasar from the statistical analyses below, since our main interest there is in the intrinsic $\mathrm{X}$-ray emission properties of quasars.

SDSS J170100.62+641209.0 $(z=2.74)$. - This quasar is the most optically luminous in the DR3 catalog and has an $\alpha_{\text {ox }}-1.91$, which differs from the predicted value by $0.7 \sigma$. After binning the data from the X-ray spectrum into 10 full-band counts per bin, we fitted the spectrum below $2 \mathrm{keV}$ to a power-law model with Galactic absorption and extrapolated the fit to higher energies; we found two consecutive bins that fall $\gtrsim 3 \sigma$ below the model near $\sim 3 \mathrm{keV}$ in the observed frame $(\sim 11 \mathrm{keV}$ in the rest frame), signs of a possible absorption feature. A spectrum taken with $X M M$ Newton (PI: F. Jansen) shows no such feature, although it is not ruled out within the uncertainties on the data points (the XMMNewton spectrum was particularly noisy due to background flaring). The binned Chandra spectrum of this quasar appears as part of Figure 5. Further observations are necessary to test the reality of this feature.

\section{DATA ANALYSIS AND RESULTS}

\subsection{X-Ray Spectral Properties}

\subsubsection{Individual-Object Analyses}

We have investigated the X-ray spectra of seven of the eight sources with Chandra observations that have $>100$ full-band counts (APM $08279+5255$ has already had its complex spectrum studied in detail in Chartas et al. 2002); below this threshold there are too few counts for statistically useful results to be derived from individual spectral fitting. The seven spectra were extracted with the CIAO routine PSEXTRACT using circular apertures of 3.0" in radius centered on the X-ray centroid of each source, with the exceptions of SDSS J0145-0945 (4.0" $0^{\prime \prime}$ used to enclose both lensed images), SDSS J1614+4704 (30.0" used due to PSF broadening at large off-axis angles), and SDSS J1701+6412 (8.0" used due to PSF broadening). Background regions were extracted using annuli of varying sizes to avoid contamination from other $\mathrm{X}$-ray sources. An annulus was not used to extract the background for SDSS J1701+6412 due to the quasar's location near the edge of the ACIS I3 CCD; instead we chose a nearby circular region $20^{\prime \prime}$ in radius that was free from other X-ray sources.

We used XSPEC version 11.3.2 (Arnaud 1996) to fit each spectrum across the full-band energy range $(0.5-8.0 \mathrm{keV})$ with a power-law model and a fixed Galactic-absorption component (Dickey \& Lockman 1990); all fits assumed solar abundances (e.g., Anders \& Grevesse 1989) and used the wabs absorption model in XSPEC. We used the $C$-statistic (Cash 1979) when modeling the unbinned data, since this method is more appropriate when fitting low-count sources than $\chi^{2}$ fitting and still remains accurate for higher numbers of counts (e.g., Nousek \& Shue 1989). All of the errors have been quoted at the $90 \%$ confidence level considering one parameter to be of interest $(\Delta C=2.71$; Avni 1976; Cash 1979). Although when using the $C$-statistic there is no value analogous to $P\left(\chi^{2} \mid \nu\right)$ with which to perform model testing, we assessed whether each model fits the data acceptably by searching for any systematic residuals. The seven objects with fitting, along with their fit parameters and statistics, appear in Table 5. In Figure 5 we present their X-ray spectra, binned at a level of 10 counts per bin for clearer presentation. Note that in Figure 5 (unlike in Table 5) we used $\chi^{2}$ fitting in order to show residuals in units of $\sigma$. The values of $\Gamma$ calculated from the band ratios (see Table 3) are consistent with those derived from the best-fit models. We also added an intrinsic, redshifted, neutralabsorption component to the model, but it did not significantly improve any of the fits.

SDSS J1701+6412 appears to show an absorption feature at $\sim 3 \mathrm{keV}$ in the observed frame (see $\S 4$ ). We used $\chi^{2}$ fitting to investigate further the significance of this feature; the $\approx 350$ fullband counts detected from this object are enough for $\chi^{2}$ fitting to be acceptable. When modeled with a power law and Galactic absorption, $P\left(\chi^{2} \mid \nu\right)=0.23$ with $\chi^{2}=34.1$ and $\nu=29$. Although this is a statistically acceptable fit, the presence of systematic residuals motivated further investigation. Performing an $F$-test, we found the addition of intrinsic absorption to the model did not significantly improve the fit. However, the addition of an absorption edge at $2.4 \mathrm{keV}$ in the observed frame $(9.0 \mathrm{keV}$ in the rest frame) significantly improved the fit at a confidence level greater than $99.6 \%\left(\Delta \chi^{2}=11.8\right.$ for 2 additional fit parameters $)$. $\mathrm{Fe} x \mathrm{xv}$ has an ionization energy of $8.8 \mathrm{keV}$, which is close to the location of the modeled edge. Further observations are required to assess better the nature of the X-ray spectral complexity in this remarkably luminous quasar.

\subsubsection{Joint Spectral Analyses}

The relatively small numbers of counts for many of the sources in our sample make it impossible to measure accurately $\Gamma$ and $N_{\mathrm{H}}$ on a source-by-source basis, so joint fitting has been used for different combinations of sources in our core sample combined with the complementary $z \gtrsim 4$ sources from $\mathrm{S} 06$ ( $\operatorname{see} \S 2.1 .2$ ). This procedure allows measurement of the average X-ray properties of sets of objects, including those with too few counts for individual spectral fitting. Only 42 sources that have Chandra observations have been used in the joint fitting. Shown in Table 6 are the results of the joint fits across the observed $0.5-8.0 \mathrm{keV}$ range, all of which exclude gravitationally lensed (since their deamplified fluxes do not meet our luminosity criterion), radio-loud, and BAL quasars. Also excluded is SDSS J1521+5202 (see $\S 4$ ), as well as sources that had less than 3 full-band counts; these include SDSS J1350+ 5716 (with 2 counts) and two of the complementary $z \gtrsim 4$ sources from S06 (BR 1117-1329, with 2 counts, and PSS 2344+0342, which was not detected in the X-ray band). We extracted the X-ray spectra of these sources using PSEXTRACT in a similar manner 


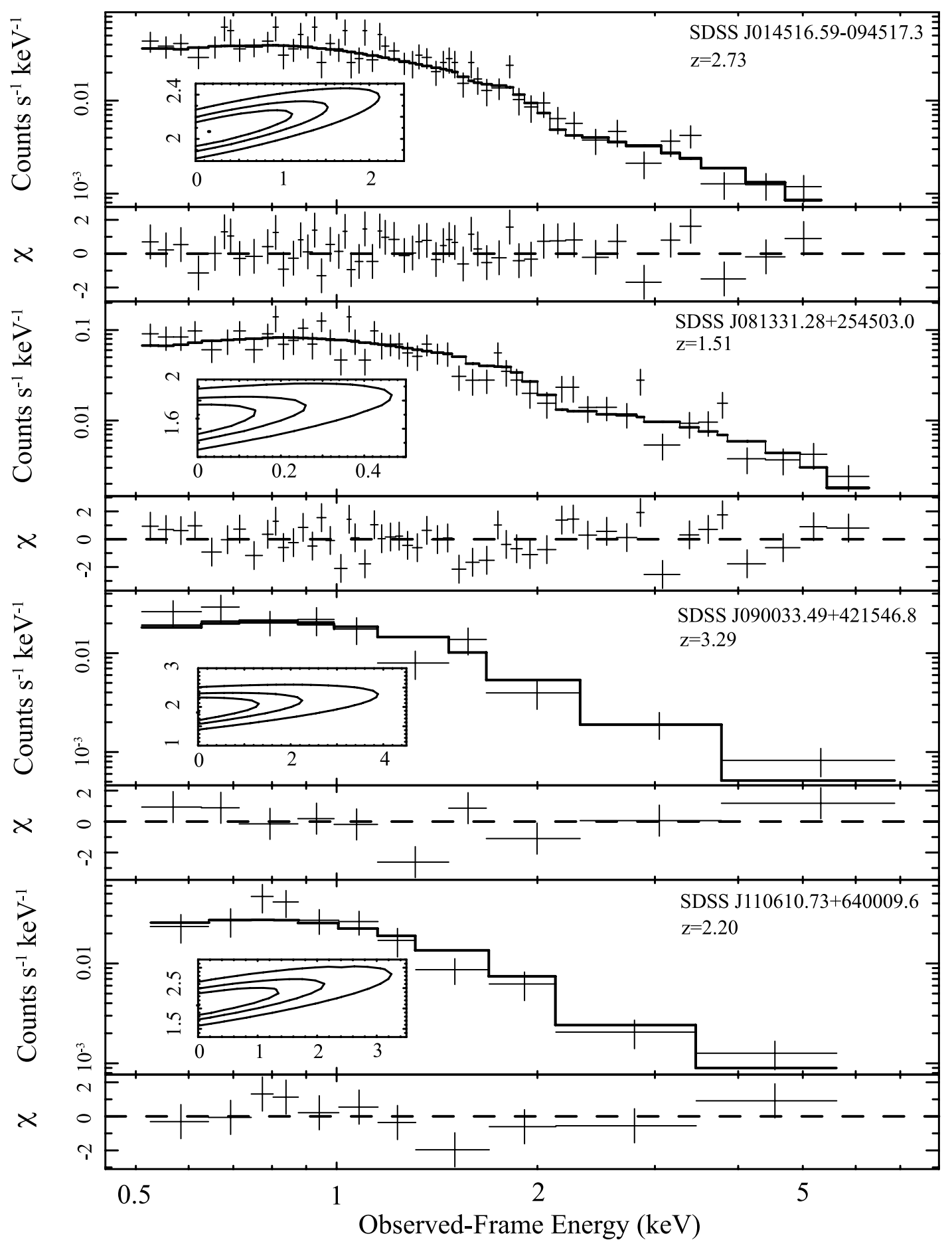

FIG. 5.- Individual Chandra X-ray spectra and residuals for seven of the quasars from our sample that have $>100$ counts. The spectra have been fitted across the full band $(0.5-8 \mathrm{keV})$. The $\chi$ residuals are in units of $\sigma$, and the inset in each panel shows a contour map of $\Gamma$ vs. intrinsic $N_{\mathrm{H}}$ (in units of $10^{22} \mathrm{~cm}^{-2}$ ) at confidence levels corresponding to $68 \%, 90 \%$, and $99 \%$.

to that described in $\S 5.1 .1$. The sets of unbinned spectra were fit using XSPEC, first with a power-law model and a Galactic absorption component, which was kept fixed during the fit, and then with an added intrinsic neutral-absorption component. All fits utilized the $C$-statistic.

We checked whether our sample was biased by objects with a high signal-to-noise ratio $(\mathrm{S} / \mathrm{N})$. We split the quasars into two groups, each having more and less than 100 full-band counts. It can be seen from Table 6 that the $\Gamma$ found for the quasars with $>100$ counts is consistent with the $\Gamma$ found for quasars with
$<100$ counts; we therefore conclude that the high $\mathrm{S} / \mathrm{N}$ spectra do not bias the overall sample (note that the total number of counts is approximately split between the two groups). The addition of an intrinsic-absorption component did not improve any of the fits; i.e., no significant amount of intrinsic absorbing material has been detected. Upper limits on intrinsic $N_{\mathrm{H}}$ appear in Table 6.

Previous studies have shown that, in general, $\Gamma$ does not evolve with redshift for luminous quasars (e.g., Page et al. 2005; Shemmer et al. 2005b, 2006b; Vignali et al. 2005). To investigate this matter further, we have performed joint fitting on sets of 


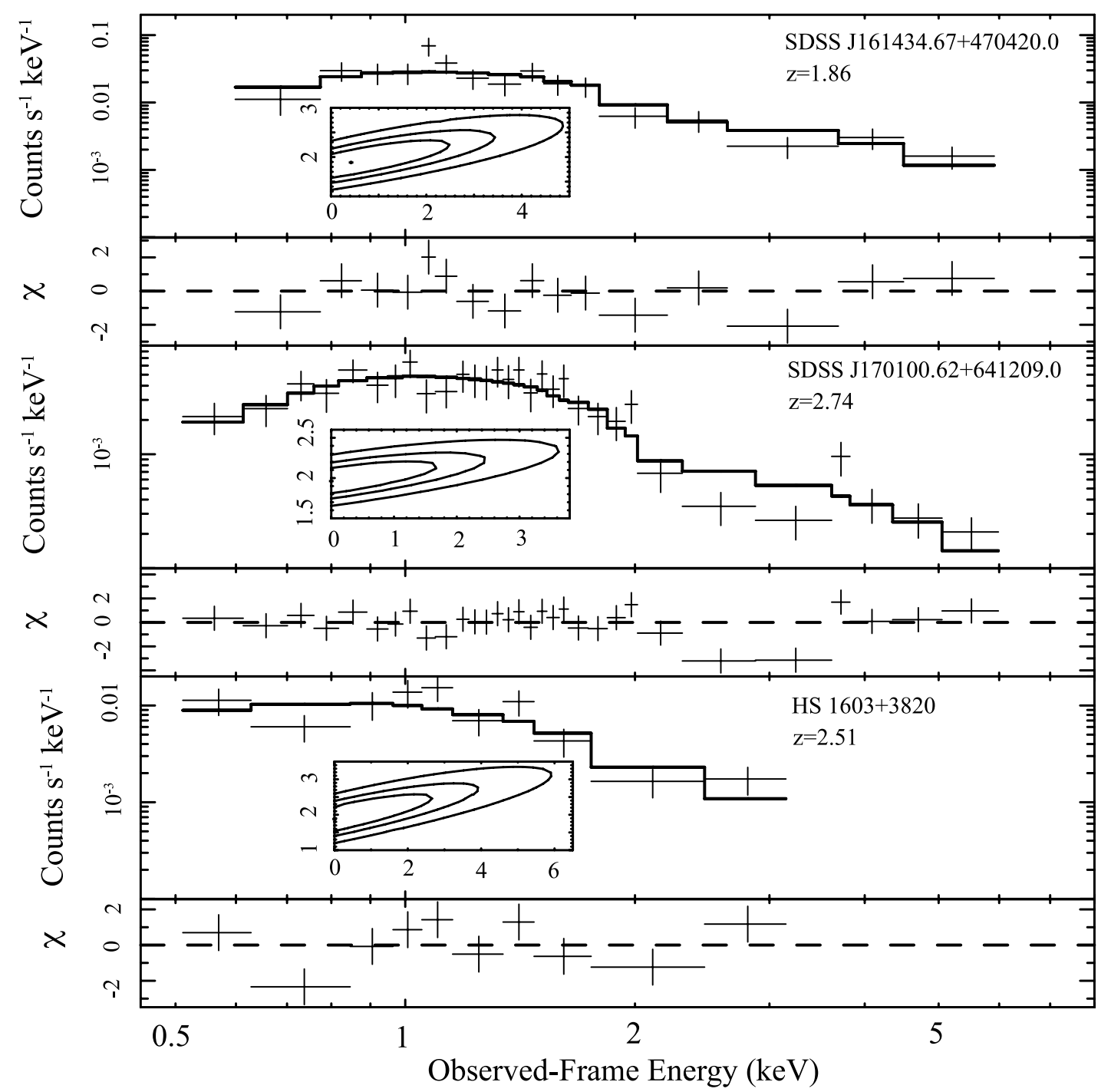

FIG. 5-Continued

TABLE 5

Individual-ObJect Spectral-Fitting Results

\begin{tabular}{|c|c|c|c|c|c|c|}
\hline Object & X-Ray Counts & $\Gamma^{\mathrm{a}}$ & $C$-Statistic ${ }^{\mathrm{a}}$ & $\Gamma^{\mathrm{b}}$ & $\begin{array}{c}N_{\mathrm{H}}^{\mathrm{b}} \\
\left(10^{22} \mathrm{~cm}^{-2}\right)\end{array}$ & $C$-Statistic ${ }^{\mathrm{b}}$ \\
\hline \multicolumn{7}{|l|}{ SDSS J: } \\
\hline 0145-0945 …….................... & 686 & $2.05_{-0.12}^{+0.12}$ & 151.7 & $2.08_{-0.15}^{+0.20}$ & $\leq 1.00$ & 151.4 \\
\hline 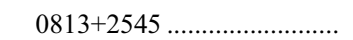 & 591 & $1.65_{-0.12}^{+0.12}$ & 195.4 & $1.65_{-0.11}^{+0.11}$ & $\leq 0.16^{\mathrm{c}}$ & 195.2 \\
\hline $0900+4215$ & 108 & $1.94_{-0.28}^{+0.12}$ & 74.5 & $1.94_{-028}^{+0.11}$ & $\leq 1.56$ & 74.5 \\
\hline $1106+6400$ & 122 & $2.01_{-0.28}^{+0.28}$ & 73.7 & $\begin{array}{l}1.44_{-0.028}^{+0.37} \\
2.01_{-0.27}^{+0.37}\end{array}$ & $\leq 1.18$ & 73.7 \\
\hline $1614+4704$ & 181 & $\begin{array}{l}2.01-0.28 \\
1.74_{-0.22}^{+0.28}\end{array}$ & 88.1 & $\begin{array}{l}2.01-0.27 \\
1.86_{-032}^{+0.35}\end{array}$ & $\leq 1.80$ & 88.0 \\
\hline $1701+6412$ & 352 & $1.91_{-0.16}^{+0.22}$ & 153.3 & $1.91_{-0.016}^{+0.32}$ & $\leq 1.47$ & 153.3 \\
\hline HS $1603+3820 \ldots \ldots \ldots \ldots \ldots \ldots \ldots$ & 116 & $2.01_{-0.28}^{+0.16}$ & 63.2 & $2.10_{-0.35}^{+0.51}$ & $\leq 1.29$ & 62.8 \\
\hline
\end{tabular}

NotE.-All fits are across observed-frame $0.5-8.0 \mathrm{keV}$ and include appropriate Galactic absorption.

${ }^{a}$ Without an intrinsic absorption component.

${ }^{\mathrm{b}}$ With an intrinsic absorption component. Upper limit on the intrinsic column density at the source redshift given in Table 1, quoted at the $90 \%$ confidence level.

${ }^{c}$ The tighter constraint on this intrinsic column density is due to the relatively low redshift $(z=1.51)$ of this object combined with its relatively large number of counts. 
TABLE 6

Joint Spectral-Fitting Results

\begin{tabular}{|c|c|c|c|c|c|c|c|c|c|}
\hline Sources Included & Number of Sources & Median Redshift & Median $M_{i}$ & Total Counts & $\Gamma^{\mathrm{a}}$ & $C$-Statistic ${ }^{\mathrm{a}}$ & $\Gamma^{\mathrm{b}}$ & $\begin{array}{c}N_{\mathrm{H}}^{\mathrm{b}} \\
\left(10^{22} \mathrm{~cm}^{-2}\right)\end{array}$ & $C$-Statistic ${ }^{\mathrm{b}}$ \\
\hline All RQQs & 42 & 4.04 & -29.36 & 1872 & $1.92_{-0.08}^{+0.09}$ & 1149.0 & $1.92_{-0.06}^{+0.10}$ & $<0.2$ & 1149.0 \\
\hline RQQs, $<100$ counts & 37 & 4.07 & -29.34 & 993 & $1.93_{-0.10}^{+0.08}$ & 695.1 & $1.93_{-0.10}^{+0.06}$ & $<0.6$ & 695.1 \\
\hline RQQs, $>100$ counts & 5 & 2.74 & -29.86 & 879 & $1.92_{-0.09}^{+0.12}$ & 454.0 & $1.91_{-0.08}^{+0.16}$ & $<0.8$ & 454.0 \\
\hline RQQs, $1<z<2$ & 4 & 1.88 & -29.41 & 343 & $1.87_{-0.14}^{+0.19}$ & 192.9 & $1.91_{-0.19}^{+0.38}$ & $<1.2$ & 192.9 \\
\hline RQQs, $2<z<3$ & 8 & 2.63 & -29.46 & 784 & $1.95_{-011}^{+0.11}$ & 446.6 & $1.95_{-011}^{+0.17}$ & $<0.8$ & 446.6 \\
\hline RQQs, $3<z<4$ & 8 & 3.37 & -29.43 & 305 & $1.90_{-0.18}^{+0.11}$ & 213.5 & $1.86_{-0.18}^{+0.11}$ & $<1.0$ & 213.7 \\
\hline RQQs, $4<z<5$ & 22 & 4.34 & -29.17 & 440 & $1.93_{-0.16}^{+0.18}$ & 295.7 & $1.87_{-0.16}^{-0.18}$ & $<0.8$ & 296.5 \\
\hline RQQs, $<100$ counts, $1<z<2 \ldots$ & 3 & 1.90 & -29.41 & 162 & $1.98^{+0.25}$ & 103.9 & $2.13^{+0.48}$ & $<2.0$ & 103.9 \\
\hline RQQs, $<100$ counts, $2<z<3$ & 5 & 2.82 & -29.39 & 194 & $1.95_{-0.23}^{+0.23}$ & 156.3 & $2.10_{-0.35}^{+0.40}$ & $<2.5$ & 156.3 \\
\hline RQQs, $<100$ counts, $3<z<4$ & 7 & 3.38 & -29.41 & 197 & $1.87_{-0.23}^{+0.23}$ & 138.9 & $1.87_{-0.22}^{+0.35}$ & $<2.1$ & 138.9 \\
\hline RQQs, $<100$ counts, $4<z<5$ & 22 & 4.34 & -29.17 & 440 & $1.93_{-0.16}^{+0.16}$ & 295.7 & $1.87_{-0.16}^{-0.22}$ & $<0.8$ & 296.5 \\
\hline 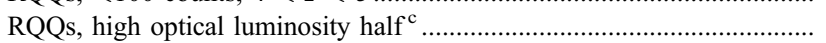 & 21 & 3.70 & -29.57 & 1151 & $1.94_{-0.09}^{+0.09}$ & 681.0 & $1.91_{-0.08}^{+0.16}$ & $<0.3$ & 681.2 \\
\hline 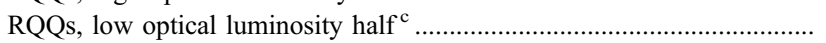 & 21 & 4.03 & -29.14 & 721 & $1.90_{-0.11}^{+0.13}$ & 468.0 & $1.97_{-0.16}^{+0.18}$ & $<1.1$ & 468.0 \\
\hline RQQs, $<100$ counts, high optical luminosity half ${ }^{\mathrm{d}} \ldots$ & 18 & 4.09 & -29.43 & 508 & $1.93_{-012}^{+0.117}$ & 358.9 & $1.91_{-012}^{+0.17}$ & $<0.7$ & 358.9 \\
\hline RQQs, $<100$ counts, low optical luminosity half ${ }^{\mathrm{d}} \ldots .$. & 19 & 4.06 & -29.02 & 485 & $1.93_{-0.15}^{+0.15}$ & 336.3 & $1.98_{-0.19}^{+0.23}$ & $<1.3$ & 336.3 \\
\hline
\end{tabular}

NotE.-Errors on $\Gamma$ and upper limits for $N_{\mathrm{H}}$ are quoted at $90 \%$ confidence levels.

${ }^{\text {a }}$ Without an intrinsic absorption component.

With an intrinsic absorption component

c The high optical luminosity and low optical luminosity halves are split above and below $\log \left(L_{2500} \AA\right)=32.1$ and have X-ray luminosities of $\log \left(\nu L_{\nu}\right)_{2 \mathrm{keV}}=44.50-45.76$ and $44.45-45.83$, respectively.

${ }^{d}$ The $<100$ count high optical luminosity and low optical luminosity halves are split above and below $\log \left(L_{2500} \AA\right)=32.1$ and have X-ray luminosities of log $\left(\nu L_{\nu}\right)_{2}$ keV $=44.50-45.83$ and $44.45-45.48$, respectively. 


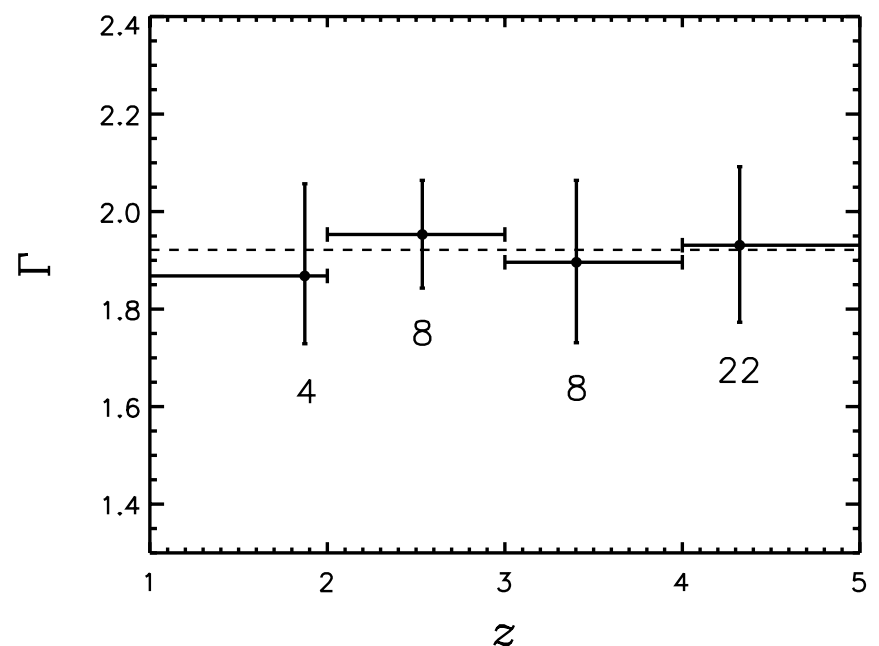

FIG. 6. - X-ray power-law photon index $(\Gamma)$ vs. redshift, binned into four integer redshift bins ranging from $z=1$ to 5 , for our sample and the complementary high-luminosity sources at $z \gtrsim 4$ from S06 (see $\S 2.1 .2$ ). The mean photon indices were derived from joint spectral fitting across the full band $(0.5-8 \mathrm{keV})$. Only sources with Chandra observations are included, while gravitationally lensed, radio-loud, and BAL quasars have been excluded (as well as SDSS J1521+5202). The vertical error bars show $90 \%$ confidence bars in each bin, and the number of sources in each bin is shown beneath the error bars; three sources with $<3$ counts (SDSS 1350+5716, BR 1117-1329, and PSS 2344+0342) did not meet our requirements for joint fitting and thus were excluded (see $\S$ 5.1.2). The horizontal error bars show the width of each bin, and the data points are marked at the median redshift in each bin. The dashed line shows the best constant-model fit, which has a value of 1.92 .

our quasars binned into four integer redshift bins ranging from $z=1$ to 5 . Our sample of the most optically luminous quasars spans the full redshift range where such objects are known to exist in the universe, $z \approx 1.5-4.5$, and it is constrained to a relatively narrow luminosity range [having a mean $\log \left(L_{2500 \AA}\right)=$ 32.2; no mean $L_{2500 \AA}$ in any integer redshift bin differs from this global mean by more than $2 \sigma$, with three of the four redshift bins differing by less than $1 \sigma$ ]. These two properties enable our sample to explore a different region of the luminosity-redshift plane than previous studies, and they minimize possible confusion between redshift-dependent and luminosity-dependent effects. For the joint fitting we used the same models described above and again found that the high-S/N sources ( $\gtrsim 100$ counts) did not bias the best-fit parameters. The best-fit parameters appear in Table 6, while a plot of $\Gamma$ versus redshift using these values is shown in Figure 6. We found no detectable change in $\Gamma$ with redshift $\left[\chi^{2}=0.5\right.$ for 3 degrees of freedom, $\left.P\left(\chi^{2} \mid \nu\right)=0.91\right]$, and basic fitting shows that the maximum allowed change in $\Gamma$ across this redshift range can be no more than $\approx 5 \%$. We have also split the sample into higher and lower optical luminosity halves and performed joint fitting; within the uncertainties the maximum allowed change of $\Gamma$ with luminosity can be no greater than $\approx 10 \%$.

At rest-frame energies below $\sim 1 \mathrm{keV}$, some quasar SEDs can deviate from a power-law due to the additional X-ray flux provided by the soft X-ray excess (e.g., Porquet et al. 2004). This additional soft X-ray emission can bias calculations of $\Gamma$ toward higher (softer) values. To see if our measurements of $\Gamma$ are biased in such a way, we redid our joint spectral analysis considering only the data above rest-frame $2 \mathrm{keV}$. Since our lower observed-frame energy limit is still $0.5 \mathrm{keV}$, this additional constraint does not affect our calculations at $z \gtrsim 3$. Implementing this additional constraint did not change our calculated values of $\Gamma$ significantly. As perhaps expected, the largest change occurred in the $z=1-2 \mathrm{bin}$.
TABLE 7

Iron $\mathrm{K} \alpha$ Line Constraints

\begin{tabular}{|c|c|c|}
\hline Sources & $\begin{array}{c}\text { Rest-Frame EW } \\
(\mathrm{eV})\end{array}$ & $\begin{array}{l}\text { Number } \\
\text { of Counts }\end{array}$ \\
\hline All RQQs, $1<z<2 \ldots$ & $\lesssim 490.6$ & 343 \\
\hline All RQQs, $2<z<3$ & $\lesssim 302.9$ & 784 \\
\hline All RQQs, $3<z<4 \ldots$ & $\lesssim 462.0$ & 305 \\
\hline All RQQs, $4<z<5^{\mathrm{b}}$ & $\lesssim 144.4$ & 440 \\
\hline RQQs, $<100$ counts, $1<z<2 \ldots \ldots \ldots \ldots \ldots . . . . .$. & $\lesssim 883.3$ & 162 \\
\hline RQQs, $<100$ counts, $2<z<3 \ldots \ldots \ldots \ldots \ldots . . . . .$. & $\lesssim 1387.2$ & 194 \\
\hline RQQs, $<100$ counts, $3<z<4 \ldots \ldots \ldots \ldots \ldots$ & $\lesssim 769.7$ & 197 \\
\hline RQQs, $<100$ counts, $4<z<5^{\mathrm{b}} \ldots \ldots \ldots \ldots \ldots$ & $\lesssim 144.4$ & 440 \\
\hline
\end{tabular}

${ }^{a}$ Calculated at the mean redshift in each bin and quoted at the $90 \%$ confidence level.

${ }^{b}$ Three of the quasars with $<3$ full-band counts were excluded.

In this redshift bin, the photon index increased from $\Gamma=1.87_{-0.14}^{+0.19}$ to $\Gamma=1.95_{-0.17}^{+0.22}$, which is well within the derived errors (and this increase goes opposite to the sense expected if soft X-ray excess emission were present). The small difference between these two methods shows that our sample is not significantly biased by excess soft X-ray emission, so we continue to use the results from joint fitting done in the observed-frame, $0.5-8.0 \mathrm{keV}$ band.

We have searched for a narrow, neutral, iron $\mathrm{K} \alpha$ line in each of the integer redshift-binned sets of spectra. No lines were detected. Upper limits on the rest-frame EWs of any such emission appear in Table 7; the rest-frame EWs were calculated at the mean redshift in each bin. These upper limits range from $\approx 150$ to $500 \mathrm{eV}$. These are not particularly tight constraints, especially when considering the high average luminosity of our sample. Quasars with higher X-ray luminosities tend to show weaker iron $\mathrm{K} \alpha$ emission lines (e.g., Page et al. 2004; Bianchi et al. 2007).

We have also checked for a Compton-reflection continuum component at $\approx 10-50 \mathrm{keV}$ in our spectra, which would be particularly apparent at high redshifts. No reflection component was found; this is not unexpected given the high luminosities and relatively low number of counts for the higher redshift sources.

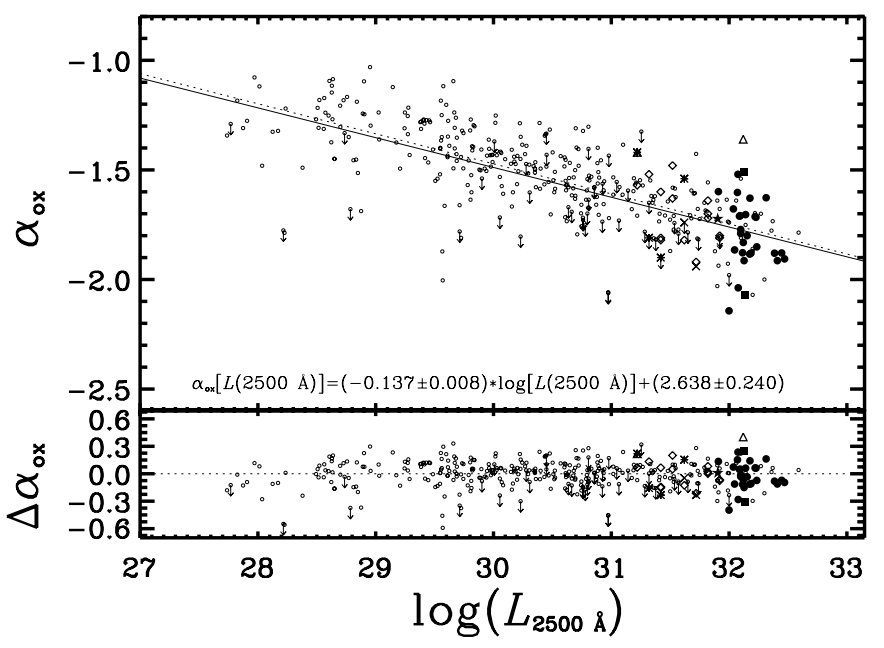

FIG. 7.-Plot of $\alpha_{\text {ox }}$ vs. $L_{2500 \AA}$ for our core sample ( filled symbols), the full S06 sample, and the Shemmer et al. (2006b) sample (open diamonds); only clean quasars have been included. Upper limits are denoted with downward-pointing arrows; all of our core-sample sources have X-ray detections. The solid (dotted) line is the best-fit relation found in this paper (S06). The lower panel shows residuals $\left(\Delta \alpha_{\mathrm{ox}}\right)$ from the S06 relation; this equation is shown at the bottom of the top panel. The most luminous object on this plot is PSS $0926+3055$, one of the complementary high-luminosity $z \gtrsim 4$ sources. 

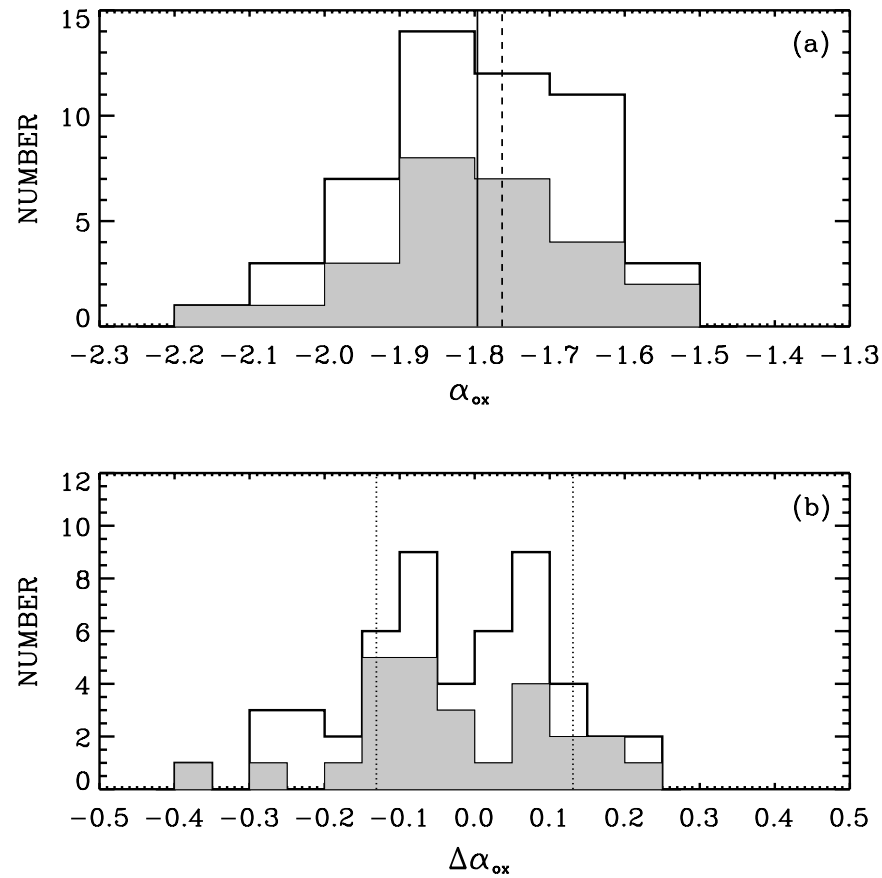

FIG. 8.- - Histograms of $(a) \alpha_{\text {ox }}$ and (b) $\Delta \alpha_{\text {ox }}$ for our 51 clean-quasar sample, with bin sizes of 0.1 and 0.05 , respectively. Our core sample is marked (light shaded histogram). The solid (dashed) line in panel $a$ marks the mean measured (predicted) $\alpha_{\text {ox }}$ of our quasar sample. In panel $b$, dotted lines mark the $1 \sigma$ range $(\sigma= \pm 0.131)$ for the distribution of $\Delta \alpha_{\text {ox }}$ for sources with $32<\log \left(L_{2500 \AA}\right)<33$, taken from Table 5 of S06.

\subsection{X-Ray-to-Optical Spectral Energy Distributions}

\subsubsection{Basic Sample Properties}

The X-ray-to-optical flux ratio for AGNs has been found to decrease at higher optical luminosities, but it does not show any clear change with redshift (e.g., Avni \& Tananbaum 1986; Wilkes et al. 1994; Strateva et al. 2005; S06 and references therein; but see Kelly et al. 2007). Using our 34 object core sample of highly luminous quasars spanning the widest possible redshift range for such objects $(z \approx 1.5-4.5)$, we further examine the $\alpha_{\text {ox }}-L_{2500} \AA$ relationship and provide constraints on $\alpha_{\mathrm{ox}}$ evolution with redshift. All of the statistical analyses presented below have excluded radio-loud, BAL, gravitationally lensed, and weak-line quasars (see below), as well as SDSS J1521+5202 (see $\S 4$ ); any group of quasars satisfying these criteria will be hereafter referred to as "clean."

Figure 7 shows $\alpha_{\text {ox }}$ versus $L_{2500 \AA}$ for our core sample combined with the full S06 sample (including the complementary high-luminosity $z \gtrsim 4$ quasars) and 14 additional $z>4$ quasars from Shemmer et al. (2006b), resulting in an X-ray detection fraction of $89 \%$. The inclusion of the 14 clean quasars from Shemmer et al. (2006b) significantly improves coverage at $z \approx 5-6$; note that the full Shemmer et al. (2006b) sample includes four weak emission line quasars, which we do not include in our analyses since the nature of these objects remains unclear. The best-fit relation from S06,

$$
\alpha_{\text {ox }}=(-0.137 \pm 0.008) \log \left(L_{2500 \AA}\right)+(2.638 \pm 0.240),
$$

is shown as a dotted line; a more detailed analysis of the best fit for the correlation between $\alpha_{\mathrm{ox}}$ and UV luminosity is given in $\S 5.2 .2$ below. Note that the addition of our core sample of 26 clean quasars to the full S06 sample increases by a factor of $\approx 2$ the number of quasars at the highest luminosities that have $\alpha_{\text {ox }}$ values.

In Figure 8 we show histograms of our $\alpha_{\text {ox }}$ distribution and the distribution of residuals from the S06 best fit $\left(\Delta \alpha_{\text {ox }}\right)$ for our 51 quasar clean sample. The 26 clean quasars from our 34 quasar core sample are marked with light shaded histograms, while the 25 complementary $z \gtrsim 4$ sources from S06 are not marked. Marked on the $\alpha_{\text {ox }}$ distribution are the measured and predicted mean $\alpha_{\mathrm{ox}}$ values, as solid and dashed lines, respectively; the predicted mean $\alpha_{\text {ox }}$ value was calculated from equation (2). Some standard statistical values for our $\alpha_{\text {ox }}$ and $\Delta \alpha_{\text {ox }}$ distributions are shown in Table 8. The mean value of $\alpha_{\mathrm{ox}}=-1.80 \pm 0.02$ for our sample agrees with the predicted value from Table 5 of S06 $(-1.788)$ to within $1 \sigma$. In the $\Delta \alpha_{\text {ox }}$ histogram, dotted lines mark the $1 \sigma$ range from the predicted S06 value (see $\S 3$, col. [13]). As a test, we have determined that it would require a reduction of $\approx 17 \%$ to the X-ray luminosities of our sources (corresponding to a change in $\alpha_{\text {ox }}$ of $\approx-0.03$ ) in order for the mean $\alpha_{\text {ox }}$ of our sample to become inconsistent with the predicted value of S06. We have also used the method of Maccacaro et al. (1988) to estimate the intrinsic dispersion of the $\alpha_{\text {ox }}$ values for our 51 non-BAL RQQs. We find a highly significant intrinsic dispersion of 0.10 ; the measured dispersion, not correcting for measurement errors, is 0.14 .

TABLE 8

$\alpha_{\text {ox }}$ and $\Delta \alpha_{\text {ox }}$ Statistical Values

\begin{tabular}{|c|c|c|c|c|c|c|}
\hline Parameter & Unweighted Mean & Measured Dispersion & Intrinsic Dispersion & First Quartile & Median & Third Quartile \\
\hline \multicolumn{7}{|l|}{ All objects (51 sources): } \\
\hline 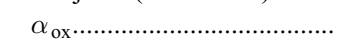 & $-1.796 \pm 0.019$ & 0.136 & 0.103 & -1.880 & -1.800 & -1.700 \\
\hline$\Delta \alpha_{\mathrm{ox}}$ & $-0.029 \pm 0.019$ & $\ldots$ & $\ldots$ & -0.110 & -0.033 & +0.072 \\
\hline \multicolumn{7}{|l|}{$1<z<2$ (4 sources) } \\
\hline$\alpha_{\mathrm{ox}} \ldots \ldots \ldots \ldots \ldots \ldots \ldots$ & $-1.805 \pm 0.060$ & 0.120 & $\ldots^{\mathrm{a}}$ & -1.880 & -1.878 & -1.865 \\
\hline$\Delta \alpha_{\mathrm{ox}}$ & $-0.044 \pm 0.056$ & $\ldots$ & $\ldots$ & -0.111 & -0.110 & -0.106 \\
\hline \multicolumn{7}{|l|}{$2<z<3$ (11 sources): } \\
\hline 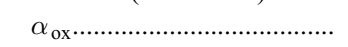 & $-1.832 \pm 0.041$ & 0.136 & 0.093 & -1.942 & -1.841 & -1.758 \\
\hline$\Delta \alpha_{\mathrm{ox}}$ & $-0.057 \pm 0.041$ & $\ldots$ & $\ldots$ & -0.120 & -0.063 & +0.009 \\
\hline \multicolumn{7}{|l|}{$3<z<4$ (11 sources): } \\
\hline 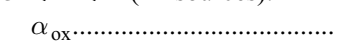 & $-1.765 \pm 0.045$ & 0.149 & 0.125 & -1.880 & -1.713 & -1.678 \\
\hline$\Delta \alpha_{\mathrm{ox}} \ldots \ldots \ldots \ldots \ldots \ldots \ldots \ldots$ & $+0.010 \pm 0.047$ & $\ldots$ & $\ldots$ & -0.079 & +0.066 & +0.084 \\
\hline \multicolumn{7}{|l|}{$4<z<5$ (25 sources): } \\
\hline$\alpha_{\mathrm{ox}} \ldots \ldots \ldots \ldots \ldots \ldots \ldots \ldots \ldots$ & $-1.795 \pm 0.026$ & 0.130 & 0.070 & -1.871 & -1.796 & -1.709 \\
\hline$\Delta \alpha_{\mathrm{ox}}$ & $-0.035 \pm 0.026$ & $\ldots$ & $\ldots$ & -0.130 & -0.022 & +0.061 \\
\hline
\end{tabular}

${ }^{\text {a }}$ Given the low number of sources in this redshift bin, we were unable to determine the intrinsic dispersion accurately. 


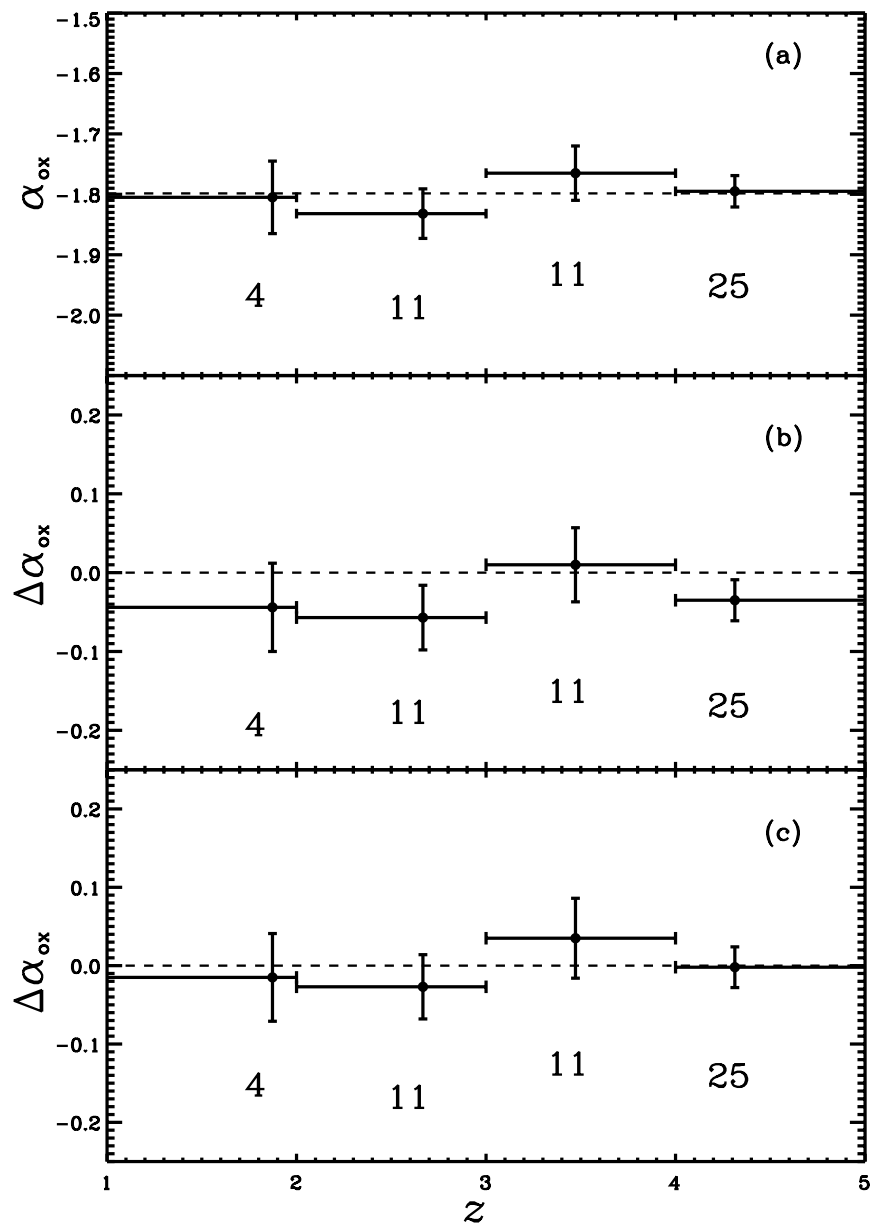

FIG. 9.-(a) Plot of $\alpha_{\text {ox }}$ vs. redshift, binned into four integer redshift bins ranging from $z=1$ to 5 . The complementary high-luminosity sources at $z \gtrsim 4$ from S06 (see $\S 2.1 .2)$ are also included in this analysis. The number of quasars in each bin is given below the error bars; these numbers differ from those in Fig. 6 due to the inclusion of ROSAT and XMM-Newton targets, as well as the three sources with $<2$ counts (SDSS 1350+5716, BR 1117-1329, and PSS 2344+0342). The vertical error bars show the standard error of the mean in each bin, while the horizontal error bars show the width of each bin; the data points are marked at the median redshift in each bin. In $(b)$ are the residuals from the best-fit relation derived in S06, while in (c) are residuals from eq. (3) of this paper, i.e., the best-fit relation found in our study. The best constant-model fit (giving $\alpha_{\mathrm{ox}}=-1.80$ ) is shown as a dashed line in $(a)$.

As a basic first test for any redshift dependence of $\alpha_{\mathrm{ox}}$, we have binned our sample of quasars, including the complementary high-luminosity $z \gtrsim 4$ sources, into integer redshift bins from $z=1$ to 5 . Recall from $\S 5.1 .2$ that the mean $L_{2500 \AA}$ in each bin does not differ from the sample mean by more than $2 \sigma$, with three of the four redshift bins differing by less than $1 \sigma$; this reduces the effect of the $\alpha_{\text {ox }}$-luminosity correlation when looking for any $\alpha_{\mathrm{ox}}-z$ correlation. The values for the mean $\alpha_{\mathrm{ox}}$ calculated in each bin, as well as the mean residuals (i.e., $\Delta \alpha_{\text {ox }}$ ) from the S06 best fit and the best fit found in this paper (see $\S 5.2 .2$, eq. [3]), are plotted against redshift in Figure 9. Error bars show the standard error of the mean in each bin. No detectable change in $\alpha_{\text {ox }}$ is evident across the full redshift range [a constant fit gives $\chi^{2}=1.3, P\left(\chi^{2} \mid \nu\right)=$ $0.74]$, and using basic fitting we have placed a constraint on any such change in $\alpha_{\text {ox }}$ to be $\lesssim 6 \%$ (corresponding to a change in the ratio of $L_{2 \mathrm{keV}}$ to $L_{2500 \AA}$ of less than a factor of 1.9).

\subsubsection{Linear-Regression Analysis}

To investigate further correlations between $\alpha_{\text {ox }}, L_{2500 \AA}, L_{2 \mathrm{keV}}$, and $z$, we have added our core sample of 26 clean quasars to the full 333 source sample of S06; we removed SDSS J1701+ 6412 from the S06 sample since it is present in both. Also included are the $14 z>4$ clean quasars from Shemmer et al. (2006b). The inclusion of our core sample of quasars, which lie in a narrow range of high luminosity $\left[\log \left(L_{2500 \AA}\right) \approx 32.0-32.5\right]$ and across a fairly wide range of redshift $(z \approx 1.5-4.5)$, allows exploration of a new region of the luminosity-redshift plane (see Fig. 10). Also shown in Figure 10 are the additional quasars from Shemmer et al. (2006b), which substantially improve coverage at $z \approx 5-6$. Ultimately, 372 quasars are included in our analysis: 26 from our core sample, 332 of the 333 from the full S06 sample, and 14 from the Shemmer et al. (2006b) sample, increasing the S06 sample size by $\approx 12 \%$. Note that we do not expect significant problematic effects from unidentified BAL quasars at $z \lesssim 1.5$ in the S06 sample (see $\S 3.3$ of Strateva et al. 2005).

To quantify the correlations found between the optical and X-ray properties, we used the Astronomy Survival Analysis software package (ASURV rev. 1.2; Isobe et al. 1990; Lavalley et al. 1992) to perform linear regressions on the data. ASURV treats censored data using the survival-analysis methods presented in Feigelson \& Nelson (1985) and Isobe et al. (1986). We used both the fully parametric EM (estimate and maximize) regression algorithm (Dempster et al. 1977) and the semiparametric BuckleyJames regression algorithm (Buckley \& James 1979) when performing linear regressions. In what follows we report the parameters derived from the EM regression, although in all cases the Buckley-James regression algorithm agreed within the errors.

We confirm and strengthen the finding in previous studies that $\alpha_{\text {ox }}$ decreases with increasing rest-frame UV luminosity. Performing linear regressions with ASURV on the combined sample of 372 quasars, we found the best-fit relation between $\alpha_{\text {ox }}$ and $L_{2500 \AA}$ to be

$$
\alpha_{\mathrm{ox}}=(-0.140 \pm 0.007) \log \left(L_{2500 \AA}\right)+(2.705 \pm 0.212) .
$$

For comparison, both our best fit as well as the S06 best fit are shown in Figure 7 as solid and dashed lines, respectively.

We also confirm a significant correlation exists between $\alpha_{\text {ox }}$ and $L_{2} \mathrm{keV}$. The best-fit parameters for this relation are

$$
\alpha_{\mathrm{ox}}=(-0.093 \pm 0.014) \log \left(L_{2 \mathrm{keV}}\right)+(0.899 \pm 0.359) .
$$

Note that the EM and Buckley-James regression algorithms are no longer strictly valid when double-censoring is present (upper limits exist on both $\alpha_{\text {ox }}$ and $L_{2} \mathrm{kev}$ in the S06 data). However, given the high X-ray detection fraction of our combined sample $(89 \%)$, we have treated the censored $L_{2} \mathrm{keV}$ data as though they were detected.

Studies measuring a relationship between X-ray and UV luminosities of the form $L_{\mathrm{X}} \propto L_{\mathrm{UV}}^{\beta}$ have found both $\beta \simeq 0.7-0.8$ (e.g., Avni \& Tananbaum 1982, 1986; Kriss \& Canizares 1985; Anderson \& Margon 1987; Wilkes et al. 1994; Vignali et al. 2003; Strateva et al. 2005; S06) and $\beta=1$ (La Franca et al. 1995). We find the best-fit parameters for the $L_{2} \mathrm{keV}-L_{2500 \AA}$ relation to be

$$
\log \left(L_{2 \mathrm{keV}}\right)=(0.636 \pm 0.018) \log \left(L_{2500 \AA}\right)+(7.055 \pm 0.553)
$$

while treating $L_{2} \mathrm{keV}$ as the dependent variable, and

$$
\log \left(L_{2 \mathrm{keV}}\right)=(0.808 \pm 0.021) \log \left(L_{2500 \AA}\right)+(1.847 \pm 0.694)
$$



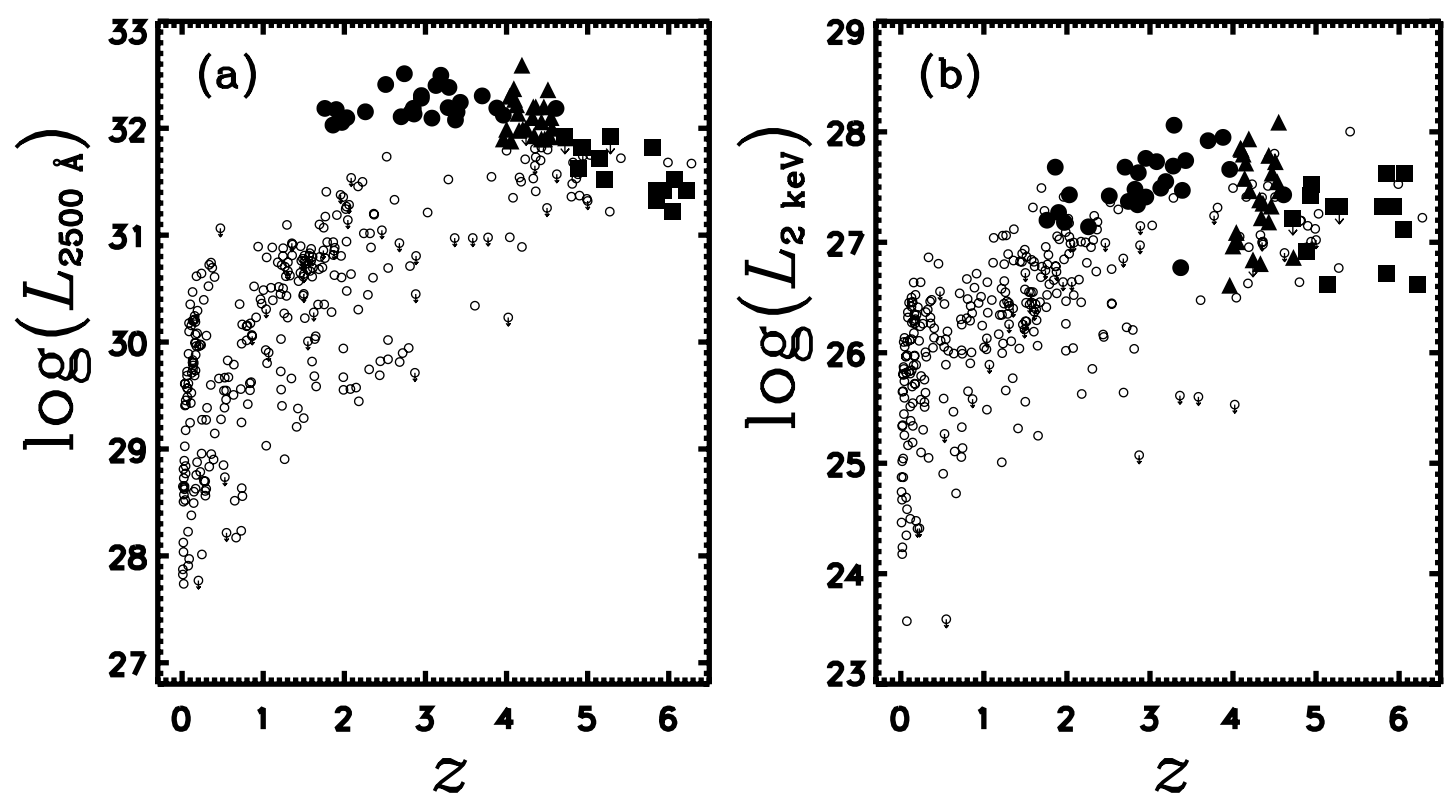

Fig. 10.- (a) $L_{2500 \AA}$ and (b) $L_{2} \mathrm{keV}$ vs. redshift for our core sample (26 filled circles) and the complementary $z \gtrsim 4$ sources from S06 (25 filled triangles). Also shown are the remaining S06 sample (307 open circles) and the Shemmer et al. (2006b) sample (14 filled squares). Gravitationally lensed, radio-loud, and BAL quasars have been removed, as well as the extreme outlier SDSS J1521+5202 (see $\S 4$ ). Note the new region of luminosity-redshift space being populated by our core sample.

while treating $L_{2 \mathrm{keV}}$ as the independent variable. Using the equations given in Table 1 of Isobe et al. (1990), we calculate the bisector of the two lines to be

$\log \left(L_{2 \mathrm{keV}}\right)=(0.709 \pm 0.010) \log \left(L_{2500 \AA}\right)+(4.822 \pm 0.627)$

This result agrees with those previous studies which found $\beta$ to be inconsistent with unity.

Finally, we used ASURV to investigate the relationship between $\alpha_{\mathrm{ox}}, L_{2500} \AA$, and $z$. We tested three different parametric forms of redshift dependence: (1) a dependence on $z$, (2) a dependence on $\log (1+z)$, and (3) a dependence on the cosmological look-back time, $\tau(z)$, in units of the present age of the universe. The best-fit parameters for these three relations are

$$
\begin{gathered}
\alpha_{\mathrm{ox}}=(-0.134 \pm 0.011) \log \left(L_{2500 \AA}\right) \\
-(0.005 \pm 0.007) z+(2.543 \pm 0.320), \\
\alpha_{\mathrm{ox}}=(-0.137 \pm 0.012) \log \left(L_{2500 \AA}\right) \\
-(0.006 \pm 0.023) \log (1+z)+(2.635 \pm 0.340), \\
\alpha_{\mathrm{ox}}=(-0.143 \pm 0.011) \log \left(L_{2500 \AA}\right) \\
-(0.001 \pm 0.003) \tau(z)+(2.824 \pm 0.350) .
\end{gathered}
$$

All three parameterizations have redshift-dependent coefficients consistent with zero; note that these equations have the same parametric form as equations (8)-(10) of Kelly et al. (2007). This finding agrees with previous studies that have found no evolution of $\alpha_{\text {ox }}$ with redshift (e.g., Strateva et al. 2005; S06), as well as our results from $\S$ 5.2.1 (also see Fig. 9).

We have followed the method described in $\S 4.4$ of S06 to compare our results directly with those from earlier studies, in particular Avni \& Tananbaum (1986), Wilkes et al. (1994), and S06. Confidence contours of $A_{O}$ [the coefficient of $\left.\log \left(L_{2500 \AA}\right)\right]$ and
$A_{\tau}$ [the coefficient of $\tau(z)$ ] were calculated using the method outlined in $\S \S 3$ and 4 of Avni \& Tananbaum (1986). Renaming $A_{z}$ as $A_{\tau}$, equation (6) of Avni \& Tananbaum (1986) becomes

$$
\begin{aligned}
& \bar{\alpha}_{\text {ox }}\left(L_{2500 \AA}, z \mid \text { X-ray loud }\right) \\
& \quad=A_{O}\left[\log \left(L_{2500 \AA}\right)-30.5\right]+A_{\tau}[\tau(z)-0.5]+A .
\end{aligned}
$$

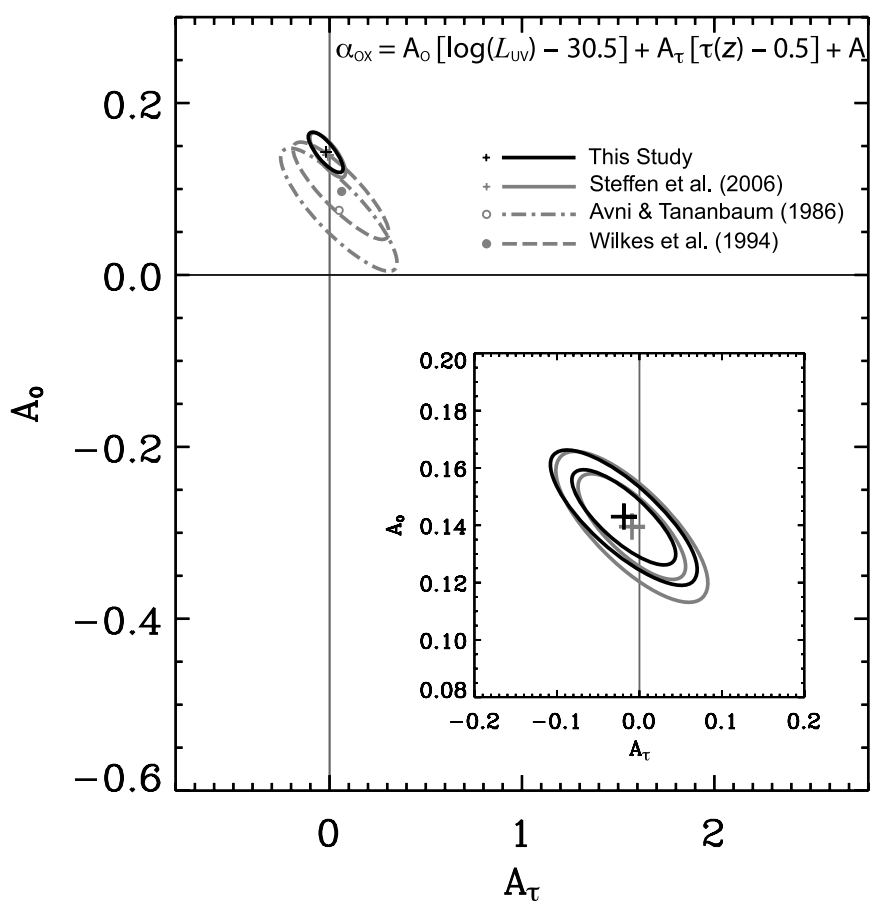

FIg. 11.-Best-fit values and $90 \%$ confidence contours for the coefficients $A_{O}$ and $A_{\tau}$ for our sample of 59 quasars combined with the S06 sample and the Shemmer et al. (2006b) sample (cross, dark solid contour), a total of 372 quasars. Also shown are best-fit values and contours for the S06 sample (cross, light solid contour), the sample of Avni \& Tananbaum (1986; open circle, dot-dashed contour), and the sample of Wilkes et al. (1994; filled circle, dashed contour). Inset: Magnified view of the $68 \%$ and $90 \%$ contours for both our sample (solid contours) and the S06 sample (light solid contours). 
We found best fit values of $\left(A_{O}, A_{\tau}, A\right)=(0.143 \pm 0.011$, $-0.016 \pm 0.041,1.556 \pm 0.009)$ for our sample. Figure 11 shows contours of $A_{O}$ and $A_{\tau}$ at both the $68 \%$ and $90 \%$ confidence levels, along with contours from previous studies. Our best-fit value has smaller confidence contours than those of previous studies and has an $A_{\tau}$ consistent with zero.

Some Monte Carlo simulations have suggested that correlations among $\alpha_{\mathrm{ox}}, L_{2500 \AA}$, and $L_{2 \mathrm{keV}}$ may arise from the effects of luminosity dispersion in optically selected, flux-limited samples (e.g., Yuan et al. 1998; Tang et al. 2007). However, these studies have usually examined the effects of luminosity dispersion over a much smaller total range in UV luminosity $\left(\Delta \log L_{2500 \AA} \sim\right.$ $2.5)$ than our full sample covers $\left(\Delta \log L_{2500} \AA 5\right)$. In $\S 3.5$ of Strateva et al. (2005) the authors estimated the dispersions of $L_{2500 \AA}$ and $L_{2 \mathrm{keV}}\left(\sigma_{\mathrm{UV}}\right.$ and $\sigma_{\mathrm{X}}$, when expressed in log units) and, using simulations, showed that the dispersions cannot be responsible for the nonunity $L_{2 \mathrm{keV}}-L_{2500 \AA}$ slope they found. They estimated that $\sigma_{\mathrm{UV}} / \sigma_{\mathrm{X}}$ is not larger than 1.4 and is plausibly $<1$ for their sample, and we expect these values to hold for our sample as well. The strength of our sample (which builds on Strateva et al. 2005) comes, in part, from the large luminosity range we cover, which is much larger than the value of the luminosity dispersion in either band. Tang et al. (2007), in their $\S 3$, do not take full advantage of the large luminosity range and examine only a subsample from S06 (and the smaller, high-redshift sample from Miyaji et al. 2006), where the luminosity range covered is only slightly larger than the dispersion. Comparison of the parametric fits calculated by Tang et al. (2007; see their Fig. 8 ) with our full sample shows very significant disagreement between the data and the fits, especially at high luminosities, where our sample of the most luminous quasars helps considerably. Tang et al. (2007), in their $\S 5$, consider selection effects using Monte Carlo simulations of a sample more similar to the full S06 sample. They assume the true slope of the $L_{2} \mathrm{keV}-L_{2500 \AA}$ relation is unity and assess how much the observed slope, $\beta$, can differ from unity. They require unrealistic combinations of $\sigma_{\mathrm{UV}}$ and $\sigma_{\mathrm{X}}$ (e.g., with $\sigma_{\mathrm{UV}} / \sigma_{\mathrm{X}} \gg 1$ ) in order to obtain values of $\beta$ as flat as the 0.709 that we measure in equation (7). While our $\beta$-value may be biased somewhat downward owing to inevitable selection effects in the presently available samples, it seems unlikely that the true slope of the $L_{2} \mathrm{keV}-L_{2500} \AA$ relation could be unity for optically selected quasar samples.

\section{CONCLUSIONS AND SUMMARY}

We have analyzed the X-ray properties of a large and statistically representative sample of the most optically luminous quasars spanning $M_{i} \approx-29.3$ to -30.2 across a redshift range of $z \approx 1.5-4.5$. Our total quasar sample consists of 59 sources, including 32 from the SDSS, two additional quasars that were missed by the SDSS selection criteria, and 25 comparably luminous $z \gtrsim 4$ quasars. All of these sources have sensitive X-ray coverage from either targeted Chandra observations or from ar- chival Chandra, ROSAT, or XMM-Newton observations; 58 of the 59 sources $(98 \%)$ have X-ray detections. For some of our analyses we have included 332 quasars from S06 and 14 quasars from Shemmer et al. (2006b). Our main results, derived for radioquiet, non-BAL quasars that are not gravitationally lensed (i.e., our "clean" quasars), are the following:

1. The mean X-ray power-law photon index for our sample of the most luminous quasars is $\Gamma=1.92_{-0.08}^{+0.09}$, consistent with values found in earlier studies.

2. Any intrinsic absorbing material for the most luminous quasars has been constrained to have a mean column density of $N_{\mathrm{H}} \lesssim$ $2 \times 10^{21} \mathrm{~cm}^{-2}$, showing that the most luminous quasars typically have little intrinsic X-ray absorption.

3. Using joint spectral fitting, we have found no significant change in $\Gamma$ with cosmic time over the redshift range $z \approx 1.5-4.5$.

4. The mean $\alpha_{\mathrm{ox}}$ value of the most luminous quasars is $\alpha_{\mathrm{ox}}=$ $-1.80 \pm 0.02$; this is in agreement with earlier studies and agrees with the predicted value from S06 [at a mean luminosity of $\left.\log \left(L_{2500 \AA}\right)=32.2\right]$ to within $1 \sigma$.

5 . In our sample there is no significant change in $\alpha_{\text {ox }}$ with redshift from $z \approx 1.5$ to 4.5 when binned and compared to a constant model, consistent with results found in some earlier studies.

6. Combining our sample with that of S06 and Shemmer et al. (2006b) results in a clean sample of 372 quasars. Using a parametric modeling method on this sample, we found that $\alpha_{\text {ox }}$ is clearly dependent on $L_{2500 \AA}$, but shows no significant dependence on redshift (for three different parameterizations of redshift dependence). The X-ray-to-optical flux ratios of quasars have not significantly evolved out to $z \sim 6$, and in particular have not significantly evolved out to $z \sim 4.5$ for the most luminous quasars.

We gratefully acknowledge the financial support of NASA grant SAO SV4-74018 (G. P. G., Principal Investigator), NASA LTSA grant NAG5-13035 (D. W. J., W. N. B., O. S., A. T. S., and D. P. S.), Chandra X-Ray Center grant GO5-6094X (D. W. J., W. N. B., and A. T. S.), and NSF grants AST 06-07634 and AST 03-07582 (D. P. S.). We thank E. O. Ofek, A. W. Rengstorf, and G. T. Richards for helpful discussions. Funding for the creation and distribution of the SDSS Archive has been provided by the Alfred P. Sloan Foundation, the Participating Institutions, the National Aeronautics and Space Administration, the National Science Foundation, the US Department of Energy, the Japanese Monbukagakusho, and the Max Planck Society. The SDSS Web site is http://www.sdss.org. The HET is a joint project of the University of Texas at Austin, the Pennsylvania State University, Stanford University, Ludwig-Maximillians-Universität München, and Georg-August-Universität Göttingen. The HET is named in honor of its principal benefactors, William P. Hobby and Robert E. Eberly.

\section{REFERENCES}

Akylas, A., Georgantopoulos, I., Georgakakis, A., Kitsionas, S., \& Hatziminaoglou, E. 2006, A\&A, 459, 693

Anders, E., \& Grevesse, N. 1989, Geochim. Cosmochim. Acta, 53, 197

Anderson, S. F., \& Margon, B. 1987, ApJ, 314, 111

Arnaud, K. A. 1996, in ASP Conf. Ser. 101, Astronomical Data Analysis Software and Systems V, ed. G. H. Jacoby \& J. Barnes (San Francisco: ASP), 17 Avni, Y. 1976, ApJ, 210, 642

Avni, Y., \& Tananbaum, H. 1982, ApJ, 262, L17 1986, ApJ, 305, 83

Bade, N., Fink, H. H., Engels, D., Voges, W., Hagen, H. J., Wisotzki, L., \& Reimers, D. 1995, A\&AS, 110, 469

Bauer, F. E., Alexander, D. M., Brandt, W. N., Schneider, D. P., Treister, E., Hornschemeier, A. E., \& Garmire, G. P. 2004, AJ, 128, 2048
Bechtold, J., et al. 2003, ApJ, 588, 119

Becker, R. H., White, R. L., \& Helfand, D. J. 1995, ApJ, 450, 559

Bianchi, S., Guainazzi, M., Matt, G., \& Fonseca Bonilla, N. 2007, A\&A, 467, L19

Brandt, W. N., Laor, A., \& Wills, B. J. 2000, ApJ, 528, 637

Buckley, J., \& James, I. 1979, Biometrika, 66, 429

Cash, W. 1979, ApJ, 228, 939

Chartas, G., Brandt, W. N., Gallagher, S. C., \& Garmire, G. P. 2002, ApJ, 579, 169

Condon, J. J., Cotton, W. D., Greisen, E. W., Yin, Q. F., Perley, R. A., Taylor, G. B., \& Broderick, J. J. 1998, AJ, 115, 1693

Croom, S. M., Smith, R. J., Boyle, B. J., Shanks, T., Miller, L., Outram, P. J., \& Loaring, N. S. 2004, MNRAS, 349, 1397 
Dai, X., Chartas, G., Eracleous, M., \& Garmire, G. P. 2004, ApJ, 605, 45 Dempster, A. P., Laird, N. M., \& Rubin, D. B. 1977, J. Royal Stat. Soc. B, 39, 1 Dickey, J. M., \& Lockman, F. J. 1990, ARA\&A, 28, 215

Djorgovski, S. G., Gal, R. R., Odewahn, S. C., de Carvalho, R. R., Brunner, R., Longo, G., \& Scaramella, R. 1998, in Wide Field Surveys in Cosmology, ed. S. Colombi \& Y. Mellier (Gif-sur-Yvette: Editions Frontieres), 89

Dobrzycki, A., Engels, D., \& Hagen, H. J. 1999, A\&A, 349, L29

Efstathiou, G., \& Rees, M. J. 1988, MNRAS, 230, 5P

Egami, E., Neugebauer, G., Soifer, B. T., Matthews, K., Ressler, M., Becklin, E. E., Murphy, T. W., Jr., \& Dale, D. A. 2000, ApJ, 535, 561

Feigelson, E. D., \& Nelson, P. I. 1985, ApJ, 293, 192

Freeman, P. E., Kashyap, V., Rosner, R., \& Lamb, D. Q. 2002, ApJS, 138, 185

Fukugita, M., Ichikawa, T., Gunn, J. E., Doi, M., Shimasaku, K., \& Schneider, D. P. 1996, AJ, 111, 1748

Gallagher, S. C., Brandt, W. N., Chartas, G., Priddey, R., Garmire, G. P., \& Sambruna, R. M. 2006, ApJ, 644, 709

Gallagher, S. C., Brandt, W. N., Laor, A., Elvis, M., Mathur, S., Wills, B. J., \& Iyomoto, N. 2001, ApJ, 546, 795

Garmire, G. P., Bautz, M. W., Ford, P. G., Nousek, J. A., \& Ricker, G. R. 2003, Proc. SPIE, 4851, 28

Gehrels, N. 1986, ApJ, 303, 336

Grupe, D., Mathur, S., Wilkes, B., \& Osmer, P. 2006, AJ, 131, 55

Hagen, H. J., Engels, D., \& Reimers, D. 1999, A\&AS, 134, 483

Irwin, M. J., Ibata, R. A., Lewis, G. F., \& Totten, E. J. 1998, ApJ, 505, 529

Isobe, T., Feigelson, E. D., Akritas, M. G., \& Babu, G. J. 1990, ApJ, 364, 104

Isobe, T., Feigelson, E. D., \& Nelson, P. I. 1986, ApJ, 306, 490

Kassiola, A., \& Kovner, I. 1992, ApJ, 388, 33

Kellermann, K. I., Sramek, R., Schmidt, M., Shaffer, D. B., \& Green, R. 1989, AJ, 98, 1195

Kelly, B. C., Bechtold, J., Siemiginowska, A., Aldcroft, T., \& Sobolewska, M. 2007, ApJ, 657, 116

Kraft, R. P., Burrows, D. N., \& Nousek, J. A. 1991, ApJ, 374, 344

Kriss, G. A., \& Canizares, C. R. 1985, ApJ, 297, 177

La Franca, F., Franceschini, A., Cristiani, S., \& Vio, R. 1995, A\&A, 299, 19

Lavalley, M., Isobe, T., \& Feigelson, E. 1992, ASP Conf. Ser. 25, Astronomical Data Analysis Software and Systems I, ed. D. M. Worrall, C. Biemesderfer, \& J. Barnes (San Francisco: ASP), 245

Lehar, J., et al. 2000, ApJ, 536, 584

Lyons, L. 1991, A Practical Guide to Data Analysis for Physical Science Students (Cambridge: Cambridge Univ. Press)

MacAlpine, G. M., \& Lewis, D. W. 1978, ApJS, 36, 587

Maccacaro, T., Gioia, I. M., Wolter, A., Zamorani, G., \& Stocke, J. T. 1988, ApJ, 326, 680

Misawa, T., Eracleous, M., Charlton, J. C., \& Tajitsu, A. 2005, ApJ, 629, 115

Miyaji, T., Hasinger, G., Lehmann, I., \& Schneider, D. P. 2006, AJ, 131, 659

Nousek, J. A., \& Shue, D. R. 1989, ApJ, 342, 1207

Oke, J. B., \& Gunn, J. E. 1983, ApJ, 266, 713

Page, K. L., O’Brien, P. T., Reeves, J. N., \& Turner, M. J. L. 2004, MNRAS, 347,316

Page, K. L., Reeves, J. N., O’Brien, P. T., \& Turner, M. J. L. 2005, MNRAS, 364,195
Piconcelli, E., Jimenez-Bailón, E., Guainazzi, M., Schartel, N., RodriguezPascual, P. M., \& Santos-Lleó, M. 2005, A\&A, 432, 15

Porquet, D., Reeves, J. N., O'Brien, P., \& Brinkmann, W. 2004, A\&A, 422, 85 Ramsey, L. W., et al. 1998, Proc. SPIE, 3352, 34

Rauch, M. 1998, ARA\&A, 36, 267

Reeves, J. N., \& Turner, M. J. L. 2000, MNRAS, 316, 234

Reimers, D., Bade, N., Schartel, N., Hagen, H. J., Engels, D., \& Toussaint, F. 1995, A\&A, 296, L49

Reimers, D., Hagen, H. J., Baade, R., Lopez, S., \& Tytler, D. 2002, A\&A, 382, L26

Rengstorf, A. W., et al. 2004, ApJ, 606, 741

Richards, G. T., et al. 2002, AJ, 123, 2945 . 2006, AJ, 131, 2766

Schneider, D. P., et al. 2005, AJ, 130, 367 (S05)

- 2007, AJ, 134, 102

Shemmer, O., Brandt, W. N., Gallagher, S. C., Vignali, C., Boller, T., Chartas, G., \& Comastri, A. 2005a, AJ, 130, 2522

Shemmer, O., Brandt, W. N., Netzer, H., Maiolino, R., \& Kaspi, S. 2006a, ApJ, 646, L29

Shemmer, O., Brandt, W. N., Vignali, C., Schneider, D. P., Fan, X., Richards, G. T., \& Strauss, M. A. 2005b, ApJ, 630, 729

Shemmer, O., et al. 2006b, ApJ, 644, 86

Springel, V., et al. 2005, Nature, 435, 629

Stark, A. A., Gammie, C. F., Wilson, R. W., Bally, J., Linke, R. A., Heiles, C., \& Hurwitz, M. 1992, ApJS, 79, 77

Steffen, A. T., Strateva, I., Brandt, W. N., Alexander, D. M., Koekemoer, A. M., Lehmer, B. D., Schneider, D. P., \& Vignali, C. 2006, AJ, 131, 2826 (S06)

Stepanian, J. A., Green, R. F., Foltz, C. B., Chaffee, F., Chavushyan, V. H., Lipovetsky, V. A., \& Erastova, L. K. 2001, AJ, 122, 3361

Strateva, I. V., Brandt, W. N., Schneider, D. P., Vanden Berk, D. G., \& Vignali, C. 2005, AJ, 130, 387

Surdej, J., Swings, J. P., Magain, P., Courvoisier, T. J. L., \& Borgeest, U. 1987, Nature, 329, 695

Surdej, J., et al. 1988, A\&A, 198, 49

Tananbaum, H., et al. 1979, ApJ, 234, L9

Tang, S. M., Zhang, S. N., \& Hopkins, P. F. 2007, MNRAS, 377, 1113

Trump, J. R., et al. 2006, ApJS, 165, 1

Turner, E. L. 1991, AJ, 101, 5

Turner, E. L., Ostriker, J. P., \& Gott, J. R., III. 1984, ApJ, 284, 1

Vanden Berk, D., et al. 2001, AJ, 122, 549 2005, AJ, 129, 2047

Vignali, C., Brandt, W. N., Schneider, D. P., Garmire, G. P., \& Kaspi, S. 2003, AJ, 125,418

Vignali, C., Brandt, W. N., Schneider, D. P., \& Kaspi, S. 2005, AJ, 129, 2519

Wilkes, B. J., Tananbaum, H., Worrall, D. M., Avni, Y., Oey, M. S., \& Flanagan, J. 1994, ApJS, 92, 53

Wolfe, A. M., Gawiser, E., \& Prochaska, J. X. 2005, ARA\&A, 43, 861

Worrall, D. M., Tananbaum, H., Giommi, P., \& Zamorani, G. 1987, ApJ, 313, 596

York, D. G., et al. 2000, AJ, 120, 1579

Yuan, W., Siebert, J., \& Brinkmann, W. 1998, A\&A, 334, 498 